\title{
Mathematical Analysis and Simulation of an Age-Structured Model of Two-Patch for Tuberculosis (TB)
}

\section{Badjo Kimba Abdoul Wahid, Saley Bisso}

Department of Mathematics and Computer Science, Abdou Moumouni University, Niamey, Niger

Email: abadjokimba@yahoo.fr, bsaley@yahoo.fr

How to cite this paper: Wahid, B.K.A. and Bisso, S. (2016) Mathematical Analysis and Simulation of an Age-Structured Model of Two-Patch for Tuberculosis (TB). Applied Mathematics, 7, 1882-1902.

http://dx.doi.org/10.4236/am.2016.715155

Received: August 8, 2016

Accepted: September 27, 2016

Published: September 30, 2016

Copyright (๑) 2016 by authors and Scientific Research Publishing Inc. This work is licensed under the Creative Commons Attribution International License (CC BY 4.0).

http://creativecommons.org/licenses/by/4.0/

\begin{abstract}
This paper studied a structured model by age of tuberculosis. A population divided into two parts was considered for the study. Each subpopulation is submitted to a program of vaccination. It was allowed the migration of vaccinated people only between the two patches. After the determination of $\mathfrak{R}(\psi)$ and $\mathfrak{R}_{0}$, the local and global stability of the disease-free equilibrium was studied. It showed the existence of three endemic equilibrium points. The theoretical results were illustrated by a numeric simulation.
\end{abstract}

\section{Keywords}

Age-Structured, Reproductive Number, Two-Patch, TB, Stability, Simulation

\section{Introduction}

Tuberculosis (TB) (short for tubercle bacillus) is a widespread, infectious disease caused by various strains of mycobacteria, usually Mycobacterium tuberculosis (MTB). Tuberculosis typically attacks the lungs, but can also affect other parts of the body [1]. To be infected bacilli must penetrate deep into the alveoli, but the contagiousness of the disease is relatively low and depends on the immune system of subjects. Individuals at highest risk are young children, adults, deficient elderly, and people living in precarious socio-economic conditions, in nursing or whose immunity is deficient (AIDS, immunosuppressive therapy ...) [2]. This is one of the most common old infectious diseases [3] [4], with about two billion people being currently infected. There are 
about nine million new cases of infection each year and two million deaths per year according to WHO estimations [3] [5]. For more information, many authors have worked on the epidemiology of tuberculosis [1]-[3] [5]-[13]. In many developing countries in general and sub-Saharan Africa particularly, TB is the leading cause of death, accounting for about two million deaths and a quarter of avoidable adult deaths [11].

It is well known that factors such as the emergence of drug resistance against tuberculosis, the growth of the incidence of HIV in recent years, as well as other diseases favor the development of Koch bacillus in the body call for improved strategies to control this deadly disease [2] [10] [14]. Last May, the World Health Assembly approved an ambitious strategy for 20 years (2016-2035) to put an end to World TB epidemic (World Day of fight against tuberculosis-March 24, 2015). In literature, several articles discussed about coinfection: TB-HIV/AIDS and the most recent is [2]. Nowadays, it is not a secret for everyone that fighting against infectious diseases is also a fight against poverty. Humans are traditionally organized into well-defined social units, such as families, tribes, villages, cities, countries or regions are good examples of patches [11] [12]. For this study, two subpopulations were considered and each was subjected to a vaccination program. However, only the vaccinated individuals can migrate from one patch to another. Despite that we have neglected the relapse rate, to avoid any risk of treated individuals' reactivation, any migration between patches was allowed. After proving that the problem is well defined and it has a unique solution if the initial condition is given, we are able to calculate the reproduction of numbers $\mathfrak{R}(\psi)$ and $\mathfrak{R}_{0}$. We have established the existence conditions for three endemic equilibrium points, and the conditions of local and global stability of the equilibrium point without disease. Finally, numerical simulations illustrate clinical outcomes. This paper is organized as follows: Section 2 introduces the two-patch model structured in age to study the dynamics of TB transmission. The existence of positive and unique solutions is demonstrated in Section 3. The point of equilibrium without disease, reproductive numbers $\mathfrak{R}(\psi)$ and $\mathfrak{R}_{0}$ are defined in the section 4 with the local and global stability of the disease-free equilibrium point. The existence of three endemic equilibrium points is proven in Section 5. Some numerical simulation results are given in Section 6. In Section 7, we have a discussion, conclusion and further work.

\section{Parameters and Mathematical Model Formulation}

Two-patch age structured model of tuberculosis was considered. The model is to split the population into two subpopulations. The recruitment is only possible in the class of susceptible and the vaccinated individuals were able to migrate between the two subpopulations. Each subpopulation is divided into five classes based on their epidemiological status: susceptible, vaccinated, latent, infectious or treated. We denote these subgroups $S_{i}(t, a), v_{i}(t, a), L_{i}(t, a), I_{i}(t, a)$ and $J_{i}(t, a)$ respectively. The birth rate of the patch $i$ is $b_{i}(a) ; \mu_{i}(a)$ and $\mu(a)$ denote the mortality rate related to the 
disease relative to the patch $i$ and the rate of natural mortality. The time and age depended of the force of infection of the subpopulation $i$ is $\lambda_{i}(t, a)$ and vaccination rate is $\psi_{i}(a) ; p_{i}\left(a, a^{\prime}\right)$ is the probability that an infective individual of age $a^{\prime}$ will have contact with and successfully infect a susceptible individual of age $a, c_{i}(a)$ is the age-specic per-capita contact/activity rate (all of these functions are assumed to be continuous and to be zero beyond some maximum age). A fraction $\phi_{i}$ of newly infected individuals of the sub-population $i$ is assumed to undergo a fast progression directly to the infectious class $I_{i}$. Rates of migration, of susceptible passage to latent infectious state and treatment are respectively $\rho_{i} ; k_{i}$ and $r_{i}$. Risk reduction rates of treatment and vaccination are $\sigma_{i}$ and $\delta_{i}$ respectively, $0 \leq \sigma_{i} \leq\left(1-\phi_{i}\right), 0 \leq \delta_{i} \leq\left(1-\phi_{i}\right)$, in this paper $i=1,2$.

The age-structured model for the transmission of TB (see Figure 1) is described by the following system of partial differential equations:

$$
\left\{\begin{array}{l}
\left(\frac{\partial}{\partial t}+\frac{\partial}{\partial a}\right) S_{1}(t, a)=b_{1}(a) N(t, a)-\left[\lambda_{1}(t, a)+\psi_{1}(a)+\mu(a)\right] S_{1}(t, a) \\
\left(\frac{\partial}{\partial t}+\frac{\partial}{\partial a}\right) L_{1}(t, a)=\lambda_{1}(t, a)\left[\left(1-\phi_{1}\right) S_{1}(t, a)+\sigma_{1} J_{1}(t, a)+\delta_{1} V_{1}(t, a)\right]-\left(k_{1}+\mu(a)\right) L_{1}(t, a) \\
\left(\frac{\partial}{\partial t}+\frac{\partial}{\partial a}\right) I_{1}(t, a)=k_{1} L_{1}(t, a)-\left(r_{1}+\mu(a)+\mu_{1}(a)\right) I_{1}(t, a)+\phi_{1} \lambda_{1}(t, a) S_{1}(t, a) \\
\left(\frac{\partial}{\partial t}+\frac{\partial}{\partial a}\right) J_{1}(t, a)=r_{1} I_{1}(t, a)-\left(\sigma_{1} \lambda_{1}(t, a)+\mu(a)\right) J_{1}(t, a) \\
\left(\frac{\partial}{\partial t}+\frac{\partial}{\partial a}\right) V_{1}(t, a)=\psi_{1}(a) S_{1}(t, a)+\rho_{2} V_{2}(t, a)-\left(\rho_{1}+\mu(a)+\delta_{1} \lambda_{1}(t, a)\right) V_{1}(t, a) \\
\left(\frac{\partial}{\partial t}+\frac{\partial}{\partial a}\right) S_{2}(t, a)=b_{2}(a) N(t, a)-\left[\lambda_{2}(t, a)+\psi_{2}(a)+\mu(a)\right] S_{2}(t, a) \\
\left(\frac{\partial}{\partial t}+\frac{\partial}{\partial a}\right) L_{2}(t, a)=\lambda_{2}(t, a)\left[\left(1-\phi_{2}\right) S_{2}(t, a)+\sigma_{2} J_{2}(t, a)+\delta_{2} V_{2}(t, a)\right]-\left(k_{2}+\mu(a)\right) L_{2}(t, a) \\
\left(\frac{\partial}{\partial t}+\frac{\partial}{\partial a}\right) I_{2}(t, a)=k_{2} L_{2}(t, a)-\left(r_{2}+\mu(a)+\mu_{2}(a)\right) I_{2}(t, a)+\phi_{2} \lambda_{2}(t, a) S_{2}(t, a) \\
\left(\frac{\partial}{\partial t}+\frac{\partial}{\partial a}\right) J_{2}(t, a)=r_{2} I_{2}(t, a)-\left(\sigma_{2} \lambda_{2}(t, a)+\mu(a)\right) J_{2}(t, a) \\
\left(\frac{\partial}{\partial t}+\frac{\partial}{\partial a}\right) V_{2}(t, a)=\psi_{2}(a) S_{2}(t, a)+\rho_{1} V_{1}(t, a)-\left(\rho_{2}+\mu(a)+\delta_{2} \lambda_{2}(t, a)\right) V_{2}(t, a)
\end{array}\right.
$$

with initial and boundary conditions:

$$
\left\{\begin{array}{l}
S_{i}(t, 0)=\int_{a_{1}}^{a_{2}} b_{i}(a) N(t, a) \mathrm{d} a \\
L_{i}(t, 0)=V_{i}(t, 0)=I_{i}(t, 0)=J_{i}(t, 0)=0 \\
S_{i}(0, a)=S_{0 i}(a) ; L_{i}(0, a)=L_{0 i}(a) ; V_{i}(0, a)=V_{0 i}(a) \\
I_{i}(0, a)=I_{0 i}(a) ; J_{i}(0, a)=J_{0 i}(a)
\end{array}\right.
$$

and $\lambda_{i}(t, a)=\beta_{i}(a) c_{i}(a) \int_{0}^{a_{+}} \frac{I_{i}\left(t, a^{\prime}\right)}{N\left(t, a^{\prime}\right)} p_{i}\left(a, a^{\prime}\right) \mathrm{d} a^{\prime}$, assume that assume that

$$
p_{i}\left(a, a^{\prime}\right)=g_{i}(a) \hat{\beta}_{i}\left(a^{\prime}\right)
$$




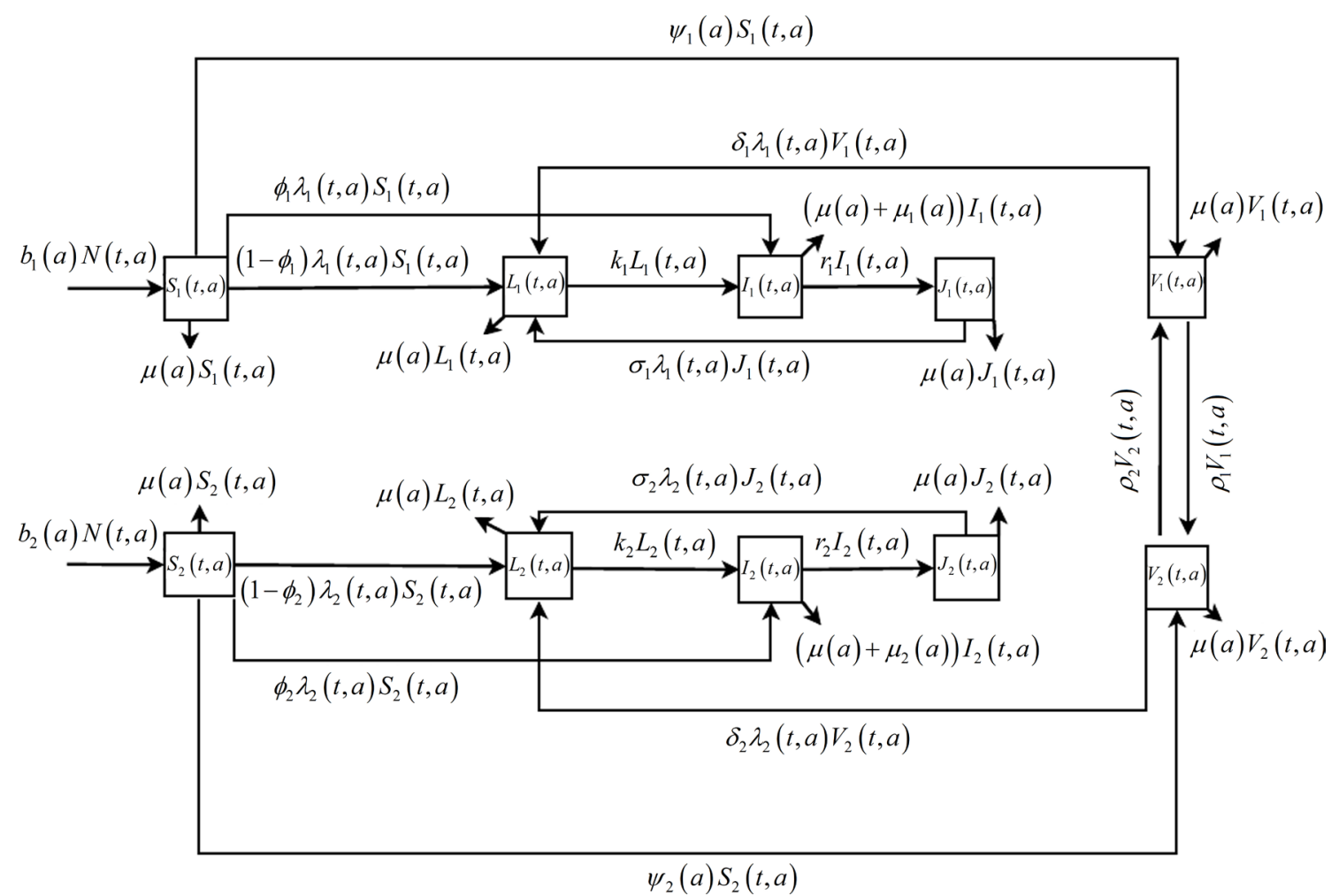

Figure 1. Flow chart of the two-patch model for tuberculosis disease transmission.

(see Greenhalgh, 1988 [15] and Dietz Schenzle, 1985 [16]), and

$$
\begin{aligned}
N(t, a)= & S_{1}(t, a)+L_{1}(t, a)+I_{1}(t, a)+J_{1}(t, a)+V_{1}(t, a) \\
& +S_{2}(t, a)+L_{2}(t, a)+I_{2}(t, a)+J_{2}(t, a)+V_{2}(t, a)
\end{aligned} .
$$

By summing equations of system (1) and (2), we obtain the following equations for the total population $N(t, a)$ :

$$
\left\{\begin{array}{l}
\left(\frac{\partial}{\partial t}+\frac{\partial}{\partial a}\right) N(t, a)=(b(a)-\mu(a)) N(t, a)-\mu_{1}(a) I_{1}(t, a)-\mu_{2}(a) I_{2}(t, a) \\
N(t, 0)=\int_{a_{1}}^{a_{2}} b(a) N(t, a) \mathrm{d} a
\end{array}\right.
$$

where $b(a)=b_{1}(a)+b_{2}(a) ; a_{1}$ and $a_{2}$ are respectively the minimum and maximum age of procreation and $a_{+}$is the maximum age of an individual, with $a_{+}<+\infty$.

Let

$$
\left\{\begin{array}{l}
s_{i}(t, a)=\frac{S_{i}(t, a)}{N(t, a)} ; l_{i}(t, a)=\frac{L_{i}(t, a)}{N(t, a)} \\
i_{i}(t, a)=\frac{I_{i}(t, a)}{N(t, a)} \\
j_{i}(t, a)=\frac{J_{i}(t, a)}{N(t, a)} ; v_{i}(t, a)=\frac{V_{i}(t, a)}{N(t, a)} .
\end{array}\right.
$$

The system (1) can be normalized as the following system: 


$$
\begin{aligned}
& \int\left(\frac{\partial}{\partial t}+\frac{\partial}{\partial a}\right) s_{1}(t, a)=b_{1}(a)-\left[\lambda_{1}(t, a)+\psi_{1}(a)+b(a)-\mu_{1}(a) i_{1}(t, a)-\mu_{2}(a) i_{2}(t, a)\right] s_{1}(t, a) \\
& \left(\frac{\partial}{\partial t}+\frac{\partial}{\partial a}\right) l_{1}(t, a)=\lambda_{1}(t, a)\left[\left(1-\phi_{1}\right) s_{1}(t, a)+\sigma_{1} j_{1}(t, a)+\delta_{1} v_{1}(t, a)\right]-\left(k_{1}+b(a)-\mu_{1}(a) i_{1}(t, a)-\mu_{2}(a) i_{2}(t, a)\right) l_{1}(t, a) \\
& \left(\frac{\partial}{\partial t}+\frac{\partial}{\partial a}\right) i_{1}(t, a)=-\left(r_{1}+\mu_{1}(a)+b(a)-\mu_{1}(a) i_{1}(t, a)-\mu_{2}(a) i_{2}(t, a)\right) i_{1}(t, a)+\phi_{1} \lambda_{1}(t, a) s_{1}(t, a)+k_{1} l_{1}(t, a) \\
& \left(\frac{\partial}{\partial t}+\frac{\partial}{\partial a}\right) j_{1}(t, a)=r_{1} i_{1}(t, a)-\left(\sigma_{1} \lambda_{1}(t, a)+b(a)-\mu_{1}(a) i_{1}(t, a)-\mu_{2}(a) i_{2}(t, a)\right) j_{1}(t, a) \\
& \left\{\left(\frac{\partial}{\partial t}+\frac{\partial}{\partial a}\right) v_{1}(t, a)=-\left(b(a)+\rho_{1}+\delta_{1} \lambda_{1}(t, a)-\mu_{1}(a) i_{1}(t, a)-\mu_{2}(a) i_{2}(t, a)\right) v_{1}(t, a)+\psi_{1}(a) s_{1}(t, a)+\rho_{2} v_{2}(t, a)\right. \\
& \left(\frac{\partial}{\partial t}+\frac{\partial}{\partial a}\right) s_{2}(t, a)=b_{2}(a)-\left[\lambda_{2}(t, a)+\psi_{2}(a)+b(a)-\mu_{1}(a) i_{1}(t, a)-\mu_{2}(a) i_{2}(t, a)\right] s_{2}(t, a) \\
& \left(\frac{\partial}{\partial t}+\frac{\partial}{\partial a}\right) l_{2}(t, a)=\lambda_{2}(t, a)\left[\left(1-\phi_{2}\right) s_{2}(t, a)+\sigma_{2} j_{2}(t, a)+\delta_{2} v_{2}(t, a)\right]-\left(k_{2}+b(a)-\mu_{1}(a) i_{1}(t, a)-\mu_{2}(a) i_{2}(t, a)\right) l_{2}(t, a) \\
& \left(\frac{\partial}{\partial t}+\frac{\partial}{\partial a}\right) i_{2}(t, a)=-\left(r_{2}+\mu_{2}(a)+b(a)-\mu_{1}(a) i_{1}(t, a)-\mu_{2}(a) i_{2}(t, a)\right) i_{2}(t, a)+\phi_{2} \lambda_{2}(t, a) s_{2}(t, a)+k_{2} l_{2}(t, a) \\
& \left(\frac{\partial}{\partial t}+\frac{\partial}{\partial a}\right) j_{2}(t, a)=r_{2} i_{2}(t, a)-\left(\sigma_{2} \lambda_{2}(t, a)+b(a)-\mu_{1}(a) i_{1}(t, a)-\mu_{2}(a) i_{2}(t, a)\right) j_{2}(t, a) \\
& \left(\frac{\partial}{\partial t}+\frac{\partial}{\partial a}\right) v_{2}(t, a)=-\left(b(a)+\rho_{2}+\delta_{2} \lambda_{2}(t, a)-\mu_{1}(a) i_{1}(t, a)-\mu_{2}(a) i_{2}(t, a)\right) v_{2}(t, a)+\psi_{2}(a) s_{2}(t, a)+\rho_{1} v_{1}(t, a)
\end{aligned}
$$

with boundary conditions

$$
s_{i}(t, 0)=\Lambda_{i} ; v_{i}(t, 0)=l_{i}(t, 0)=i_{i}(t, 0)=j_{i}(t, 0)=0
$$

with $\Lambda_{1}+\Lambda_{2}=1$. The problem is well-posedness, the methode of proof is the same used in [8].

\section{Existence of Positive Solutions}

In this section we will prove that the system (5) has a unique positive solution, and to achieve this we will write the system (5) in compact form (abstract Cauchy problem).

Consider the Banach space $X$ defined by $X=\left(L^{1}\left(0, a_{+}\right)\right)^{10}$, endowed with the norm

$$
\|\varphi\|=\sum_{i=1}^{2} \sum_{j=1}^{5}\left\|\varphi_{i j}\right\|
$$

where

$$
\begin{aligned}
& \varphi(a)=\left(\varphi_{11}(a), \varphi_{12}(a), \varphi_{13}(a), \varphi_{14}(a), \varphi_{15}(a), \varphi_{21}(a), \varphi_{22}(a), \varphi_{23}(a), \varphi_{24}(a), \varphi_{25}(a)\right)^{\mathrm{T}} \in X \\
& \text { and }\|\cdot\| \text { is the norm of } L^{1}\left(0, a_{+}\right) . \text {Let } \\
& \Omega=\left\{\left(s_{1}, l_{1}, i_{1}, j_{1}, v_{1}, s_{2}, l_{2}, i_{2}, j_{2}, v_{2}\right) \in X_{+} \backslash 0 \leq s_{1}+l_{1}+i_{1}+j_{1}, v_{1}+s_{2}+l_{2}+i_{2}+j_{2}+v_{2} \leq 1\right\}
\end{aligned}
$$

The state space of system (5), where $X_{+}=\left(L_{+}^{1}\left(0, a_{+}\right)\right)^{10}$, and $L_{+}^{1}\left(0, a_{+}\right)$denotes the positive cone of $L^{1}\left(0, a_{+}\right)$. Let $A$ be a linear operator defined by

$$
(A \varphi)(a)=\left(A_{11}, A_{12}, A_{13}, A_{14}, A_{15}, A_{21}, A_{22}, A_{23}, A_{24}, A_{25}\right)^{\mathrm{T}} \text {. }
$$

To determine the components $A_{i j}$, we neglect terms of order two and those which 
are not multiplied by $s_{i}, l_{i}, i_{i}, j_{i}$ or $v_{i}$ in system (5) (see [17]), we obtain:

$$
(a)\left\{\begin{array}{l}
\frac{\partial}{\partial t} i_{1}(t, a)=-\frac{\partial}{\partial a} i_{1}(t, a)-\left(r_{1}+\mu_{1}(a)+b(a)\right) i_{1}(t, a)+k_{1} l_{1}(t, a) \\
\frac{\partial}{\partial t} j_{1}(t, a)=-\frac{\partial}{\partial a} j_{1}(t, a)+r_{1} i_{1}(t, a)-b(a) j_{1}(t, a) \\
\frac{\partial}{\partial t} v_{1}(t, a)=-\frac{\partial}{\partial a} v_{1}(t, a)-\left(b(a)+\rho_{1}\right) v_{1}(t, a)+\psi_{1}(a) s_{1}(t, a)+\rho_{2} v_{2}(t, a) \\
\frac{\partial}{\partial t} s_{2}(t, a)=-\frac{\partial}{\partial a} s_{2}(t, a)-\left[\psi_{2}(a)+b(a)\right] s_{2}(t, a) \\
\frac{\partial}{\partial t} l_{2}(t, a)=-\frac{\partial}{\partial a} l_{2}(t, a)-\left(k_{2}+b(a)\right) l_{2}(t, a) \\
\frac{\partial}{\partial t} i_{2}(t, a)=-\frac{\partial}{\partial a} i_{2}(t, a)-\left(r_{2}+\mu_{i}(a)+b(a)\right) i_{2}(t, a)+k_{2} l_{2}(t, a) \\
\frac{\partial}{\partial t} j_{2}(t, a)=-\frac{\partial}{\partial a} j_{2}(t, a)+r_{2} i_{2}(t, a)-b(a) j_{2}(t, a) \\
\frac{\partial}{\partial t} v_{2}(t, a)=-\frac{\partial}{\partial a} v_{2}(t, a)-\left(b(a)+\rho_{2}\right) v_{2}(t, a)+\psi_{2}(a) s_{2}(t, a)+\rho_{1} v_{1}(t, a)
\end{array}\right.
$$

After replacing $s_{1}, l_{1}, i_{1}, j_{1}, v_{1}, s_{2}, l_{2}, i_{2}, j_{2}$ and $v_{2}$ by $\varphi_{11}(a), \varphi_{12}(a)$, $\varphi_{13}(a), \varphi_{14}(a), \varphi_{15}(a), \varphi_{21}(a), \varphi_{22}(a), \varphi_{23}(a), \varphi_{24}(a), \varphi_{25}(a)$ in the system (a) respectively, the coordinates of $A_{i j}$ are obtained from straight expressions (note that each

$\left.A_{i j}=\left(f\left(\varphi_{11}\right), f\left(\varphi_{12}\right), f\left(\varphi_{13}\right), f\left(\varphi_{14}\right), f\left(\varphi_{15}\right), f\left(\varphi_{21}\right), f\left(\varphi_{22}\right), f\left(\varphi_{23}\right), f\left(\varphi_{24}\right), f\left(\varphi_{25}\right)\right)\right)$ with respect to $\varphi_{i j}$ are given by:

$$
\left\{\begin{array}{l}
A_{12}=\left(0,-\frac{\mathrm{d}}{\mathrm{d} a} \varphi_{12}-\left(b(a)+k_{1}\right) \varphi_{12}, 0,0,0,0,0,0,0,0\right) \\
A_{13}=\left(0, k_{1} \varphi_{12},-\frac{\mathrm{d}}{\mathrm{d} a} \varphi_{13}-\left(r_{1}+\mu_{1}(a)+b(a)\right) \varphi_{13}, 0,0,0,0,0,0,0\right) \\
A_{14}=\left(0,0, r_{1} \varphi_{13},-\frac{\mathrm{d}}{\mathrm{d} a} \varphi_{14}-b(a) \varphi_{14}, 0,0,0,0,0,0\right) \\
A_{15}=\left(\psi,(a) \varphi_{11}, 0,0,0,-\frac{\mathrm{d}}{\mathrm{d} a} \varphi_{15}-\left(\rho_{1}+b(a)\right) \varphi_{15}, 0,0,0,0, \rho_{2} \varphi_{25}\right) \\
A_{21}=\left(0,0,0,0,0,-\frac{\mathrm{d}}{\mathrm{d} a} \varphi_{21}-\left(\psi \psi_{2}(a)+b(a)\right) \varphi_{21}, 0,0,0,0\right) \\
A_{22}=\left(0,0,0,0,0,0,-\frac{\mathrm{d}}{\mathrm{d} a} \varphi_{22}-\left(b(a)+k_{2}\right) \varphi_{22}, 0,0,0\right) \\
A_{23}=\left(0,0,0,0,0,0, k_{2} \varphi_{22},-\frac{\mathrm{d}}{\mathrm{d} a} \varphi_{23}-\left(r_{2}+\mu_{2}(a)+b(a)\right) \varphi_{23}, 0,0\right) \\
A_{24}=\left(0,0,0,0,0,0,0, r_{2} \varphi_{23},-\frac{\mathrm{d}}{\mathrm{d} a} \varphi_{24}-b(a) \varphi_{24}, 0\right) \\
A_{25}=\left(0,0,0,0, \rho_{1} \varphi_{15}, \psi \psi_{2}(a) \varphi_{21}, 0,0,0,-\frac{\mathrm{d}}{\mathrm{d} a} \varphi_{25}-\left(\rho_{2}+b(a)\right) \varphi_{25}\right)
\end{array} .\right.
$$

With

$$
\varphi(a)=\left(\varphi_{11}(a), \varphi_{12}(a), \varphi_{13}(a), \varphi_{14}(a), \varphi_{15}(a), \varphi_{21}(a), \varphi_{22}(a), \varphi_{23}(a), \varphi_{24}(a), \varphi_{25}(a)\right)^{\mathrm{T}} \in D(A)
$$


where $D(A)$ is the domain given by:

$$
D(A)=\left\{\varphi \in X \backslash \varphi_{i j} \in A C\left[0, a_{+}\right), \varphi(0)=\left(\Lambda_{1}, 0,0,0,0,0, \Lambda_{2}, 0,0,0,0\right)^{\mathrm{T}}\right\} .
$$

And $A C\left[0, a_{+}\right)$denotes the set of absolutely continuous functions on $\left[0, a_{+}\right)$. We also define a nonlinear operator $F: X \rightarrow X$ by:

$$
(F \varphi)(a)=\left(\begin{array}{c}
b_{1}(a)-\left(\left(Q_{1} \varphi_{13}\right)(a)\right) \varphi_{11}+\left(\mu_{1}(a) \varphi_{13}+\mu_{2}(a) \varphi_{23}\right) \varphi_{11} \\
\left(\left(Q_{1} \varphi_{13}\right)(a)\right)\left(\left(1-\varphi_{1}\right) \varphi_{11}+\sigma_{1} \varphi_{14}+\delta_{1} \varphi_{15}\right)+\left(\mu_{1}(a) \varphi_{13}+\mu_{2}(a) \varphi_{23}\right) \varphi_{12} \\
\varphi_{1}\left(\left(Q_{1} \varphi_{13}\right)(a)\right) \varphi_{11}+\left(\mu_{1}(a) \varphi_{13}+\mu_{2}(a) \varphi_{23}\right) \varphi_{13} \\
\left(\mu_{1}(a) \varphi_{13}+\mu_{2}(a) \varphi_{23}\right)-\delta_{1}\left(\left(Q_{1} \varphi_{13}\right)(a)\right) \varphi_{14} \\
\left(\mu_{1}(a) \varphi_{13}+\mu_{2}(a) \varphi_{23}\right)-\sigma_{1}\left(\left(Q_{1} \varphi_{13}\right)(a)\right) \varphi_{15} \\
b_{2}(a)-\left(\left(Q_{2} \varphi_{23}\right)(a)\right) \varphi_{21}+\left(\mu_{1}(a) \varphi_{13}+\mu_{2}(a) \varphi_{23}\right) \varphi_{21} \\
\left(\left(Q_{2} \varphi_{23}\right)(a)\right)\left(\left(1-\varphi_{2}\right) \varphi_{21}+\sigma_{2} \varphi_{24}+\delta_{2} \varphi_{25}\right)+\left(\mu_{1}(a) \varphi_{13}+\mu_{2}(a) \varphi_{23}\right) \varphi_{22} \\
\varphi_{2}\left(\left(Q_{2} \varphi_{23}\right)(a)\right) \varphi_{21}+\left(\mu_{1}(a) \varphi_{13}+\mu_{2}(a) \varphi_{23}\right) \varphi_{23} \\
\left(\mu_{1}(a) \varphi_{13}+\mu_{2}(a) \varphi_{23}\right)-\delta_{2}\left(\left(Q_{2} \varphi_{23}\right)(a)\right) \varphi_{24} \\
\left(\mu_{1}(a) \varphi_{13}+\mu_{2}(a) \varphi_{23}\right)-\sigma_{2}\left(\left(Q_{2} \varphi_{23}\right)(a)\right) \varphi_{25}
\end{array}\right)
$$

where $Q_{i}$ is a bounded linear operator on $L^{1}\left(0, a_{+}\right)$given by

$$
\left(Q_{i} f\right)(a)=c_{i}(a) \beta_{i}(a) g_{i}(a) \int_{0}^{a_{+}} \hat{\beta}_{i}\left(a^{\prime}\right) f\left(a^{\prime}\right) \mathrm{d} a^{\prime} .
$$

Let

$$
u(t)=\left(s_{1}(., t), l_{1}(., t), i_{1}(., t), j_{1}(., t), v_{1}(., t), s_{2}(., t), l_{2}(., t), i_{2}(., t), j_{2}(., t), v_{2}(., t)\right)
$$

thus, we can rewrite the system (5) as an abstract Cauchy problem:

$$
\left\{\begin{array}{l}
\frac{\mathrm{d}}{\mathrm{d} t} u(t)=A u(t)+F(u(t)) \\
u(0)=u_{0}
\end{array}\right.
$$

where

$$
u_{0}(a)=\left(s_{01}(a), l_{01}(a), i_{01}(a), j_{01}(a), v_{01}(a), s_{02}(a), l_{02}(a), i_{02}(a), j_{02}(a), v_{02}(a)\right)^{\mathrm{T}} .
$$

According to these results we have the following results (see [17]-[19]):

Lemma 1. The operator $F$ is continuously Fréchet differentiable on $X$.

Lemma 2. The operator A generates a $C_{0}$-semigroup of the bounded linear operators $\mathrm{e}^{t A}$ and the space $\Omega$ is positively invariant by $\mathrm{e}^{t A}$.

Theorem 1. For each $u_{0} \in X_{+}$there are a maximal interval of existence $\left[0, t_{\max }\right)$ and a unique continuous mild solution $u\left(t, u_{0}\right) \in X_{+}, t \in\left[0, t_{\max }\right)$ for (12) such that

$$
u(t)=u_{0} \mathrm{e}^{t A}+\int_{0}^{t} \mathrm{e}^{A(t-\xi)} F(u(\xi)) \mathrm{d} \xi
$$

Proof. The proof of this theorem can be found in [18]-[20].

\section{The Disease-Free Steady State}

\subsection{Determination of the Disease-Free Equilibrium}

A steady state $\left(s_{1}(a), l_{1}(a), i_{1}(a), j_{1}(a), v_{1}(a), s_{2}(a), l_{2}(a), i_{2}(a), j_{2}(a), v_{2}(a)\right)$ of sys- 
tem (5) must satisfy the following time-independent system of ordinary differential equations:

$$
\left\{\begin{aligned}
\frac{\mathrm{d}}{\mathrm{d} a} s_{1}(a)= & b_{1}(a)-\left[\beta_{1}(a) c_{1}(a) g_{1}(a) \Gamma_{1}+\psi_{1}(a)+b(a)-\mu_{1}(a) i_{1}(a)-\mu_{2}(a) i_{2}(a)\right] s_{1}(a) \\
\frac{\mathrm{d}}{\mathrm{d} a} l_{1}(a)= & \beta_{1}(a) c_{1}(a) g_{1}(a) \Gamma_{1}\left[\left(1-\phi_{1}\right) s_{1}(a)+\delta_{1} v_{1}(a)+\sigma_{1} j_{1}(a)\right] \\
& -\left(b(a)+k_{1}-\mu_{1}(a) i_{1}(a)-\mu_{2}(a) i_{2}(a)\right) l_{1}(a) \\
\frac{\mathrm{d}}{\mathrm{d} a} i_{1}(a)= & k_{1} l_{1}(a)+\phi_{1} \beta_{1}(a) c_{1}(a) g_{1}(a) \Gamma_{1} s_{1}(a)-\left[r_{1}+b(a)+\mu_{1}(a)-\mu_{1}(a) i_{1}(a)-\mu_{2}(a) i_{2}(a)\right] i_{1}(a) \\
\frac{\mathrm{d}}{\mathrm{d} a} j_{1}(a)= & r_{1} i_{1}(a)-\left(\sigma_{1} \beta_{1}(a) c_{1}(a) g_{1}(a) \Gamma_{1}+b(a)-\mu_{1}(a) i_{1}(a)-\mu_{2}(a) i_{2}(a)\right) j_{1}(a) \\
\frac{\mathrm{d}}{\mathrm{d} a} v_{1}(a)= & \psi_{1}(a) s_{1}(a)+\rho_{2} v_{2}(a)-\left(\delta_{1} \beta_{1}(a) c_{1}(a) g_{1}(a) \Gamma_{1}+b(a)-\mu_{1}(a) i_{1}(a)-\mu_{2}(a) i_{2}(a)+\rho_{1}\right) v_{1}(a) \\
\frac{\mathrm{d}}{\mathrm{d} a} s_{2}(a)= & b_{2}(a)-\left[\beta_{2}(a) c_{2}(a) g_{2}(a) \Gamma_{2}+\psi_{2}(a)+b(a)-\mu_{1}(a) i_{1}(a)-\mu_{2}(a) i_{2}(a)\right] s_{2}(a) \\
\frac{\mathrm{d}}{\mathrm{d} a} l_{2}(a)= & \beta_{2}(a) c_{2}(a) g_{2}(a) \Gamma_{2}\left[\left(1-\phi_{2}\right) s_{2}(a)+\delta_{2} v_{2}(a)+\sigma_{2} j_{2}(a)\right] \\
& -\left(b(a)+k_{2}-\mu_{1}(a) i_{1}(a)-\mu_{2}(a) i_{2}(a)\right) l_{2}(a) \\
\frac{\mathrm{d}}{\mathrm{d} a} i_{2}(a)= & k_{2} l_{2}(a)+\phi_{2} \beta_{2}(a) c_{2}(a) g_{2}(a) \Gamma_{2} s_{2}(a)-\left[r_{2}+b(a)+\mu_{2}(a)-\mu_{1}(a) i_{1}(a)-\mu_{2}(a) i_{2}(a)\right] i_{2}(a) \\
\frac{\mathrm{d}}{\mathrm{d} a} j_{2}(a)= & r_{2} i_{2}(a)-\left(\sigma_{2} \beta_{2}(a) c_{2}(a) g_{2}(a) \Gamma_{2}+b(a)-\mu_{1}(a) i_{1}(a)-\mu_{2}(a) i_{2}(a)\right) j_{2}(a) \\
\frac{\mathrm{d}}{\mathrm{d} a} v_{2}(a)= & \psi_{2}(a) s_{2}(a)+\rho_{1} v_{1}(a)-\left(\delta_{2} \beta_{2}(a) c_{2}(a) g_{2}(a) \Gamma_{2}+b(a)-\mu_{1}(a) i_{1}(a)-\mu_{2}(a) i_{2}(a)+\rho_{2}\right) v_{2}(a) \\
\Gamma_{i}=\int_{0}^{a_{+}} \hat{\beta}_{i}(a) i_{i}(a) \mathrm{d} a &
\end{aligned}\right.
$$

with initial value conditions

$$
s_{i}(0)=\Lambda_{i} ; l_{i}(0)=i_{i}(0)=j_{i}(0)=v_{i}(0)=0 .
$$

Therefore, we obtain the disease-free steady state

$$
\left\{\begin{array}{l}
s_{i}^{0}(a)=\Lambda_{i} \mathrm{e}^{-\int_{0}^{a}\left(b(\tau)+\psi_{i}(\tau)\right) \mathrm{d} \tau}+\int_{0}^{a} \mathrm{e}^{-\int_{\eta}^{a}\left(b(\tau)+\psi_{i}(\tau)\right) \mathrm{d} \tau} b_{i}(\eta) \mathrm{d} \eta \\
v_{i}^{0}(a)=\Lambda_{i}-s_{i}^{0}(a) ; l_{i}^{0}(a)=i_{i}^{0}(a)=j_{i}^{0}(a)=0
\end{array} .\right.
$$

\subsection{Calculation of the Reproduction Numbers $\mathfrak{R}(\psi)-\mathfrak{R}_{0}$}

To study the stability of the disease-free steady state, we denote the perturbations of system by

$$
\left\{\begin{array}{l}
s_{i}(t, a)=\bar{s}_{i}(t, a)+s_{i}^{0}(a) \\
l_{i}(t, a)=\bar{l}_{i}(t, a)+l_{i}^{0}(a) \\
i_{i}(t, a)=\bar{i}_{i}(t, a)+i_{i}^{0}(a) \\
j_{i}(t, a)=\bar{j}_{i}(t, a)+j_{i}^{0}(a) \\
v_{i}(t, a)=\bar{v}_{i}(t, a)+v_{i}^{0}(a)
\end{array} .\right.
$$


The perturbations satisfy the following equations:

$$
\begin{aligned}
& \int\left(\frac{\partial}{\partial t}+\frac{\partial}{\partial a}\right) \bar{s}_{1}(t, a)=-\left[\bar{\gamma}_{1}(t) \beta_{1}(a) c_{1}(a) g_{1}(a)-\mu_{1}(a) \bar{i}_{1}(t, a)-\mu_{2}(a) \bar{i}_{2}(t, a)\right] s_{1}^{0}(a) \\
& -\left(b(a)+\psi_{1}(a)\right) \bar{s}_{1}(t, a) \\
& \left(\frac{\partial}{\partial t}+\frac{\partial}{\partial a}\right) \bar{l}_{1}(t, a)=-\left(b(a)+k_{1}\right) \bar{l}_{1}(t, a) \\
& +\bar{\gamma}_{1}(t) \beta_{1}(a) c_{1}(a) g_{1}(a)\left[\left(1-\phi_{1}\right) s_{1}^{0}(a)+\delta_{1} v_{1}^{0}(a)\right] \\
& \left(\frac{\partial}{\partial t}+\frac{\partial}{\partial a}\right) \bar{i}_{1}(t, a)=k_{1} \bar{l}_{1}(t, a)+\phi_{1} \beta_{1}(a) c_{1}(a) g_{1}(a) \bar{\gamma}_{1}(t) s_{1}^{0}(a) \\
& -\left(r_{1}+\mu_{1}(a)+b(a)\right) \bar{i}_{1}(t, a) \\
& \left(\frac{\partial}{\partial t}+\frac{\partial}{\partial a}\right) \bar{j}_{1}(t, a)=r_{1} \bar{i}_{1}(t, a)-b(a) \bar{j}_{1}(t, a) \\
& \left(\frac{\partial}{\partial t}+\frac{\partial}{\partial a}\right) \bar{v}_{1}(t, a)=\psi_{1}(a) \bar{s}_{1}(t, a)+\rho_{2} \bar{v}_{2}(t, a)-\left(\rho_{1}+b(a)\right) \bar{v}_{1}(t, a) \\
& -\left(\delta_{1} \beta_{1}(a) c_{1}(a) g_{1}(a) \bar{\gamma}(t)-\mu_{1}(a) \bar{i}_{1}(t, a)-\mu_{2}(a) \bar{i}_{2}(t, a)\right) v_{1}^{0}(a) \\
& \left\{\left(\frac{\partial}{\partial t}+\frac{\partial}{\partial a}\right) \bar{s}_{2}(t, a)=-\left[\bar{\gamma}_{2}(t) \beta_{2}(a) c_{2}(a) g_{2}(a)-\mu_{1}(a) \bar{i}_{1}(t, a)-\mu_{2}(a) \bar{i}_{2}(t, a)\right] s_{2}^{0}(a)\right. \\
& -\left(b(a)+\psi_{2}(a)\right) \bar{s}_{2}(t, a) \\
& \left(\frac{\partial}{\partial t}+\frac{\partial}{\partial a}\right) \bar{l}_{2}(t, a)=-\left(b(a)+k_{2}\right) \bar{l}_{2}(t, a) \\
& +\bar{\gamma}_{2}(t) \beta_{2}(a) c_{2}(a) g_{2}(a)\left[\left(1-\phi_{2}\right) s_{2}^{0}(a)+\delta_{2} v_{2}^{0}(a)\right] \\
& \left(\frac{\partial}{\partial t}+\frac{\partial}{\partial a}\right) \bar{i}_{2}(t, a)=k_{2} \bar{l}_{2}(t, a)+\phi_{2} \beta_{2}(a) c_{2}(a) g_{2}(a) \bar{\gamma}_{2}(t) s_{2}^{0}(a) \\
& -\left(r_{2}+\mu_{2}(a)+b(a)\right) \bar{i}_{2}(t, a) \\
& \left(\frac{\partial}{\partial t}+\frac{\partial}{\partial a}\right) \bar{j}_{2}(t, a)=r_{2} \bar{i}_{2}(t, a)-b(a) \bar{j}_{2}(t, a) \\
& \left(\frac{\partial}{\partial t}+\frac{\partial}{\partial a}\right) \bar{v}_{2}(t, a)=\psi_{2}(a) \bar{s}_{2}(t, a)+\rho_{1} \bar{v}_{1}(t, a)-\left(\rho_{2}+b(a)\right) \bar{v}_{2}(t, a) \\
& -\left(\delta_{2} \beta_{2}(a) c_{2}(a) g_{2}(a) \bar{\gamma}_{2}(t)-\mu_{1}(a) \bar{i}_{1}(t, a)-\mu_{2}(a) \bar{i}_{2}(t, a)\right) v_{2}^{0}(a) \\
& \bar{\gamma}_{i}(t)=\int_{0}^{a_{+}} \hat{\beta}_{i}(a) \bar{i}_{i}(t, a) \mathrm{d} a
\end{aligned}
$$

with boundary conditions:

$$
\bar{s}_{i}(t, 0)=\bar{l}_{i}(t, 0)={\overline{i_{i}}}_{i}(t, 0)=\bar{j}_{i}(t, 0)=\bar{v}_{i}(t, 0)=0,
$$

we consider the exponential solutions of system (16) of the form:

$$
\left\{\begin{array}{l}
\bar{s}_{i}(t, a)=\bar{s}_{i}(a) \mathrm{e}^{\lambda t} ; \bar{l}_{i}(t, a)=\bar{l}_{i}(a) \mathrm{e}^{\lambda t} \\
\bar{v}_{i}(t, a)=\bar{v}_{i}(a) \mathrm{e}^{\lambda t} \\
\bar{i}_{i}(t, a)=\bar{j}_{i}(a) \mathrm{e}^{\lambda t} ; \bar{j}_{i}(t, a)=\bar{j}_{i}(a) \mathrm{e}^{\lambda t}
\end{array} .\right.
$$

The system (16) becomes: 


$$
\left\{\begin{array}{l}
\frac{\mathrm{d}}{\mathrm{d} a} \bar{s}_{1}(a)=-\left(b(a)+\psi_{1}(a)+\lambda\right) \bar{s}_{1}(a)-\left[\bar{\Gamma}_{1} \beta_{1}(a) c_{1}(a) g_{1}(a)-\mu_{1}(a) \bar{i}_{1}(a)-\mu_{2}(a) \bar{i}_{2}(a)\right] s_{1}^{0}(a) \\
\frac{\mathrm{d}}{\mathrm{d} a} \bar{l}_{1}(a)=-\left(b(a)+k_{1}+\lambda\right) \bar{l}_{1}(a)+\bar{\Gamma}_{1} \beta_{1}(a) c_{1}(a) g_{1}(a)\left[\left(1-\phi_{1}\right) s_{1}^{0}(a)+\delta_{1} v_{1}^{0}(a)\right] \\
\frac{\mathrm{d}}{\mathrm{d} a} \bar{i}_{1}(a)=\phi_{1} \beta_{1}(a) c_{1}(a) g_{1}(a) \bar{\Gamma}_{1}+k_{1} \bar{l}_{1}(a)-\left(r_{1}+\mu_{1}(a)+b(a)+\lambda\right) \bar{i}_{1}(a) \\
\frac{\mathrm{d}}{\mathrm{d} a} \bar{j}_{1}(a)=r_{1} \bar{i}_{1}(a)-(b(a)+\lambda) \bar{j}_{1}(a) \\
\frac{\mathrm{d}}{\mathrm{d} a} \bar{v}_{1}(a)=-\left(\rho_{1}+b(a)+\lambda\right) \bar{v}_{1}(a)-\left(\delta_{1} \bar{\Gamma} \beta_{1}(a) c_{1}(a) g_{1}(a)-\mu_{1}(a) \bar{i}_{1}(a)-\mu_{2}(a) \bar{i}_{2}(a)\right) v_{1}^{0}(a)+\psi_{1}(a) \bar{s}(a)+\rho_{2} \bar{v}_{2}(a) \\
\frac{\mathrm{d}}{\mathrm{d} a} \bar{S}_{2}(a)=-\left(b(a)+\psi_{2}(a)+\lambda\right) \bar{s}_{2}(a)-\left[\bar{\Gamma}_{2} \beta_{2}(a) c_{2}(a) g_{2}(a)-\mu_{1}(a) \bar{i}_{1}(a)-\mu_{2}(a) \bar{i}_{2}(a)\right] s_{2}^{0}(a) \\
\frac{\mathrm{d}}{\mathrm{d} a} \bar{l}_{2}(a)=-\left(b(a)+k_{2}+\lambda\right) \bar{l}_{2}(a)+\bar{\Gamma}_{2} \beta_{2}(a) c_{2}(a) g_{2}(a)\left[\left(1-\phi_{2}\right) s_{2}^{0}(a)+\delta_{2} v_{2}^{0}(a)\right] \\
\frac{\mathrm{d}}{\mathrm{d} a} \bar{i}_{2}(a)=\phi_{2} \beta_{2}(a) c_{2}(a) g_{2}(a) \bar{\Gamma}_{2}+k_{2} \bar{l}_{2}(a)-\left(r_{2}+\mu_{2}(a)+b(a)+\lambda\right) \bar{i}_{2}(a) \\
\frac{\mathrm{d}}{\mathrm{d} a} \bar{j}_{2}(a)=r_{2} \bar{i}_{2}(a)-(b(a)+\lambda) \bar{j}_{2}(a) \\
\frac{\mathrm{d}}{\mathrm{d} a} \bar{v}_{2}(a)=-\left(\rho_{2}+b(a)+\lambda\right) \bar{v}_{2}(a)-\left(\delta_{2} \bar{\Gamma} \beta_{2}(a) c_{2}(a) g_{2}(a)-\mu_{1}(a) \bar{i}_{1}(a)-\mu_{2}(a) \bar{i}_{2}(a)\right) v_{2}^{0}(a)+\psi_{2}(a) \bar{s}_{2}(a)+\rho_{1} \bar{v}_{1}(a) \\
\bar{\Gamma}_{i}=\int_{0}^{a_{+}} \hat{\beta}_{i}(a) \bar{i}_{i}(a) \mathrm{d} a
\end{array}\right.
$$

with boundary conditions:

$$
\bar{S}_{i}(0)=\bar{l}_{i}(0)=\bar{i}_{i}(0)=\bar{j}_{i}(0)=\bar{v}_{i}(0)=0
$$

Let

$$
N_{\psi_{i}}(a)=\left(1-\phi_{i}\right) s_{i}^{0}(a)+\delta_{i} v_{i}^{0}(a) .
$$

From Equation (18), we obtain:

$$
\begin{gathered}
\bar{l}_{i}(a)=\bar{\Gamma}_{i} \int_{0}^{a} \mathrm{e}^{-\int_{\eta}^{a}\left(b(\tau)+k_{i}+\lambda\right) \mathrm{d} \tau} \beta_{i}(\eta) c_{i}(\eta) g_{i}(\eta) N_{\psi_{i}}(\eta) \mathrm{d} \eta \\
\overline{i_{i}}(a)=\int_{0}^{a} \mathrm{e}^{-\int_{\eta}^{a}\left(b(\tau)+r_{i}+\lambda+\mu_{i}(a)\right) \mathrm{d} \tau}\left(k_{i} \bar{l}_{i}(\eta)+\bar{\Gamma}_{i} \phi_{i} \beta_{i}(\eta) c_{i}(\eta) g_{i}(\eta)\right) \mathrm{d} \eta .
\end{gathered}
$$

Hence, by Equations ((20) and (21)) after changing order of integration, we obtain:

$$
\bar{i}_{i}(a)=\bar{\Gamma}_{i} \int_{0}^{a} \mathrm{e}^{-\int_{\eta}^{a}\left(b(\tau)+\mu_{i}(\tau)+r_{i}+\lambda\right) \mathrm{d} \tau} \beta_{i}(\eta) c_{i}(\eta) g_{i}(\eta)\left[\phi_{i} s_{i}^{0}(\eta)+k_{i} N_{\psi_{i}}(\eta) \int_{\eta}^{a} \mathrm{e}^{-\int_{\alpha}^{\eta}\left(\mu_{i}(\tau)+r_{i}-k_{i}\right) \mathrm{d} \tau} \mathrm{d} \alpha\right] \mathrm{d} \eta .
$$

Injecting (22) in the expression of $\bar{\Gamma}_{i}$, and dividing both sides the expression by $\bar{\Gamma}_{i}$ (since $\bar{\Gamma}_{i} \neq 0$ ), we get the characteristic equation:

$$
1=\int_{0}^{a_{+}} \hat{\beta}_{i}(a) \int_{0}^{a} \mathrm{e}^{-\int_{\eta}^{a}\left(b(\tau)+\mu_{i}(\tau)+r_{i}+\lambda\right) \mathrm{d} \tau} \beta_{i}(\eta) c_{i}(\eta) g_{i}(\eta)\left[\phi_{i} s_{i}^{0}(\eta)+k_{i} N_{\psi_{i}}(\eta) \int_{\eta}^{a} \mathrm{e}^{-\int_{\alpha}^{\eta}\left(\mu_{i}(\tau)+r_{i}-k_{i}\right) \mathrm{d} \tau} \mathrm{d} \alpha\right] \mathrm{d} \eta \mathrm{d} a .
$$

Denote the right-hand side of Equation (23) by $G(\lambda)$ i.e.:

$$
G_{i}(\lambda)=\int_{0}^{a_{+}} \hat{\beta}_{i}(a) \int_{0}^{a} \mathrm{e}^{-\int_{\eta}^{a}\left(b(\tau)+\mu_{i}(\tau)+r_{i}+\lambda\right) \mathrm{d} \tau} \beta_{i}(\eta) c_{i}(\eta) g_{i}(\eta)\left[\phi_{i} s_{i}^{0}(\eta)+k_{i} N_{\psi_{i}}(\eta) \int_{\eta}^{a} \mathrm{e}^{-\int_{\alpha}^{\eta}\left(\mu_{i}(\tau)+r_{i}-k_{i}\right) \mathrm{d} \tau} \mathrm{d} \alpha\right] \mathrm{d} \eta \mathrm{d} a .
$$

We define the net reproductive number as $\mathfrak{R}^{i}(\psi)=G_{i}(0)$, i.e. 


$$
\mathfrak{R}^{i}\left(\psi_{i}\right)=\int_{0}^{a_{+}} \hat{\beta}_{i}(a) \int_{0}^{a} \mathrm{e}^{-\int_{\eta}^{a}\left(b(\tau)+\mu_{i}(\tau)+r_{i}\right) \mathrm{d} \tau} \beta_{i}(\eta) c_{i}(\eta) g_{i}(\eta)\left[\phi_{i} s_{i}^{0}(\eta)+k_{i} N_{\psi_{i}}(\eta) \int_{\eta}^{a} \mathrm{e}^{-\int_{\alpha}^{\eta}\left(\mu_{i}(\tau)+r_{i}-k_{i}\right) \mathrm{d} \tau} \mathrm{d} \alpha\right] \mathrm{d} \eta \mathrm{d} a .
$$

We can obtain an expression for $\mathfrak{R}_{0}^{i}$ in a similar way as the derivation of $\mathfrak{R}^{i}(\psi)$ by considering Equation (1) without vaccination; i.e., by assuming that $\psi_{i}(a) \equiv 0$ and neglecting the equation of vaccinated. It can be shown that $\mathfrak{R}_{0}^{i}=\mathfrak{R}^{i}(0)$ which is called the basic reproductive number (when a purely susceptible population is considered) (see [8]).

$$
\mathfrak{R}_{0}^{i}=\Lambda_{i} \int_{0}^{a_{+}} \hat{\beta}_{i}(a) \int_{0}^{a} \mathrm{e}^{-\int_{\eta}^{a}\left(b(\tau)+\mu_{i}(\tau)+r_{i}\right) \mathrm{d} \tau} \beta_{i}(\eta) c_{i}(\eta) g_{i}(\eta)\left[\phi_{i}+k_{i}\left(1-\phi_{i}\right) \int_{\eta}^{a} \mathrm{e}^{-\int_{\alpha}^{\eta}\left(\mu_{i}(\tau)+r_{i}-k_{i}\right) \mathrm{d} \tau} \mathrm{d} \alpha\right] \mathrm{d} \eta \mathrm{d} a
$$

Let

$$
\mathfrak{R}(\psi)=\max _{i} \mathfrak{R}^{i}\left(\psi_{i}\right) \text { and } \mathfrak{R}_{0}=\max _{i} \mathfrak{R}_{0}^{i}
$$

\subsection{Local Stability of the Disease-Free Equilibrium}

Theorem 2. The infection-free steady-state (5) is locally asymptotically stable (1.a.s.) if $\mathfrak{R}(\psi)<1$ and unstable if $\mathfrak{R}(\psi)>1$.

Proof. Noticing that

$$
G_{i}^{\prime}(\lambda)<0 ; \lim _{\lambda \rightarrow+\infty} G_{i}(\lambda)=0 ; \lim _{\lambda \rightarrow-\infty} G_{i}(\lambda)=+\infty .
$$

We know that Equation (23) has a unique negative real solution $\lambda^{*}$ if, and only if, $G_{i}(0)<1$, hence, $\mathfrak{R}^{i}\left(\psi_{i}\right)<1$ (Also, Equation (23) has a unique positive (zero) real solution if $\mathfrak{R}^{i}\left(\psi_{i}\right)>1 \quad\left(\mathfrak{R}^{i}\left(\psi_{i}\right)=1\right)$. To show that $\lambda^{*}$ is the dominant real part of roots of $G_{i}(\lambda)$, we let $\lambda=x+i y$ be an arbitrary complex solution to Equation (23). Note that

$$
1=G_{i}(\lambda)=\left|G_{i}(x+i y)\right| \leq G_{i}(x),
$$

indicating that $R_{e}(\lambda) \leq \lambda^{*}$. It follows that the infection-free steady state is l.a.s. if $\mathfrak{R}(\psi)<1$, and unstable if $\mathfrak{R}(\psi)>1$.

In this corollary, we have the three cases of the unstability of the disease free equilibrium.

Corollary 1. 1) whenever $\mathfrak{R}^{1}\left(\psi_{1}\right)<1$ and $\mathfrak{R}^{2}\left(\psi_{2}\right)>1$, the disease free is locally asymptotically stable in the first patch and unstable in the second.

2) whenever $\mathfrak{R}^{1}\left(\psi_{1}\right)>1$ and $\mathfrak{R}^{2}\left(\psi_{2}\right)<1$, the disease free is unstable in the first patch and locally asymptotically stable in the second.

3) whenever $\mathfrak{R}^{1}\left(\psi_{1}\right)>1$ and $\mathfrak{R}^{2}\left(\psi_{2}\right)>1$, the disease free is unstable in the two patches.

\subsection{Global Stability of the Disease-Free Equilibrium}

Since $\mu_{i}(a)$ and $i_{i}(t, a)$ are bounded, there exists a positive constant $R_{c}$ that satisfies

$$
0 \leq \int_{\eta}^{a} \sum_{i=1}^{2} \mu_{i}(\tau) i_{i}(t-a+\tau, \tau) \mathrm{d} \tau \leq R_{c}
$$

Corollary 2. Assume that $r_{i}+\mu_{i}(\tau) \leq k_{i}$, then we have 


$$
\begin{aligned}
& {\left[\mathrm{e}^{-\int_{\eta}^{a}\left(r_{i}+\mu_{i}(\tau)+b(\tau)\right) \mathrm{d} \tau} \phi_{i} s_{i}(t-a+\eta, \eta)+k_{i}\left(\sigma_{i} j_{i}(t-a+\eta, \eta)+\delta_{i} v_{i}(t-\eta+a, \eta)\right.\right.} \\
& \left.+\left(1-\phi_{i}\right) s_{i}(t-a+\eta, \eta) \mathrm{e}^{-\int_{\eta}^{a}\left(k_{i}+b(\tau)\right) \mathrm{d} \tau} \int_{\eta}^{a} \mathrm{e}^{-\int_{\xi}^{a}\left(r_{i} \mu_{i}(\tau)-k_{i}\right) \mathrm{d} \tau} \mathrm{d} \xi\right] \\
& \leq \Lambda_{i} \mathrm{e}^{-\int_{\eta}^{a}\left(b(\tau)+\mu_{i}(\tau)+r_{i}\right) \mathrm{d} \tau}\left[\phi_{i}+k_{i}\left(1-\phi_{i}\right) \int_{\eta}^{a} \mathrm{e}^{-\int_{\xi}^{\eta}\left(\mu_{i}(\tau)+r_{i}-k_{i}\right) \mathrm{d} \tau} \mathrm{d} \xi\right]
\end{aligned}
$$

Theorem 3. The disease-free equilibrium of system (5) is globally asymptotically stable if $\mathfrak{R}_{0}<1$ and $R_{c}<\ln \left(\frac{1}{\mathfrak{R}_{0}}\right)$.

Proof. The proof consist to show that

$i_{i}(t, a) \rightarrow 0 ; \quad j_{i}(t, a) \rightarrow 0 ; \quad l_{i}(t, a) \rightarrow 0 ;$

$s_{i}(t, a) \rightarrow s_{i}^{0}(a)$ and $v_{i}(t, a) \rightarrow \Lambda_{i}-s_{i}^{0}(a)$, when $t \rightarrow+\infty$.

Integrating system (5) along characteristic lines we get

$$
\begin{aligned}
l_{i}(t, a)= & \int_{0}^{a} \mathrm{e}^{-\int_{\eta}^{a}\left(b(\tau)-\sum_{i=1}^{2} \mu_{i}(\tau) i_{i}(t-a+\tau, \tau) \mathrm{d} \tau+k_{i}\right) \mathrm{d} \tau} \beta_{i}(\eta) c_{i}(\eta) g_{i}(\eta) \lambda_{i}(t-a+\eta) \\
& \times\left[\sigma_{i} j_{i}(t-a+\eta, \eta)+\delta_{i} v_{i}(t-\eta+a, \eta)+\left(1-\phi_{i}\right) s_{i}(t-a+\eta, \eta)\right] \mathrm{d} \eta, a<t \\
i_{i}(t, a)= & \int_{0}^{a} \mathrm{e}^{-\int_{\xi}^{a}\left(b(\tau)-\sum_{i=1}^{2} \mu_{i}(\tau) \dot{i}_{i}(t-a+\tau, \tau)+r_{i}+\mu_{i}(\tau)\right) \mathrm{d} \tau} \\
& \times\left[\phi_{i} \beta_{i}(\xi) c_{i}(\xi) g_{i}(\xi) \lambda_{i}(t-a+\xi)+k_{i} l(t-a+\xi, \xi)\right] \mathrm{d} \xi, a<t
\end{aligned}
$$

Injecting (27) in (28), and changing order of integration, we obtain:

$$
\begin{aligned}
i_{i}(t, a)= & \int_{0}^{a} \mathrm{e}^{\int_{\eta}^{a} \sum_{i=1}^{2} \mu_{i}(\tau) i_{i}(t-a+\tau, \tau) \mathrm{d} \tau} \beta_{i}(\eta) c_{i}(\eta) g_{i}(\eta) \lambda_{i}(t-a+\eta) \\
& \times\left[\mathrm{e}^{-\int_{\eta}^{a}\left(r_{i}+\mu_{i}(\tau)+b(\tau)\right) \mathrm{d} \tau} \phi_{i} s_{i}(t-a+\eta, \eta)+k_{i}\left(\sigma_{i} j_{i}(t-a+\eta, \eta)+\delta_{i} v_{i}(t-\eta+a, \eta)\right.\right. \\
& \left.\left.+\left(1-\phi_{i}\right) s_{i}(t-a+\eta, \eta) \mathrm{e}^{-\int_{\eta}^{a}\left(k_{i}+b(\tau)\right) \mathrm{d} \tau} \int_{\eta}^{a} \mathrm{e}^{-\int_{\xi}^{\eta}\left(r_{i}+\mu_{i}(\tau)-k_{i}\right) \mathrm{d} \tau} \mathrm{d} \xi\right)\right] \mathrm{d} \eta
\end{aligned}
$$

Injecting (29) in $\lambda_{i}(t)$, and changing order of integration, we obtain:

$$
\begin{aligned}
\lambda_{i}(t)= & \int_{0}^{a_{+}} \hat{\beta}_{i}(a) \int_{0}^{a} \mathrm{e}^{\int_{\eta}^{a} \sum_{i=1}^{2} \mu_{i}(\tau) i_{i}(t-a+\tau, \tau) \mathrm{d} \tau} \beta_{i}(\eta) c_{i}(\eta) g_{i}(\eta) \lambda_{i}(t-a+\eta) \\
& \times\left[\mathrm{e}^{-\int_{\eta}^{a}\left(r_{i}+\mu_{i}(\tau)+b(\tau)\right) \mathrm{d} \tau} \phi_{i} s_{i}(t-a+\eta, \eta)+k_{i}\left(\sigma_{i} j_{i}(t-a+\eta, \eta)+\delta_{i} v_{i}(t-\eta+a, \eta)\right.\right. \\
& \left.\left.+\left(1-\phi_{i}\right) s_{i}(t-a+\eta, \eta) \mathrm{e}^{-\int_{\eta}^{a}\left(k_{i}+b(\tau)\right) \mathrm{d} \tau} \int_{\eta}^{a} \mathrm{e}^{-\int_{\xi}^{\eta}\left(r_{i}+\mu_{i}(\tau)-k_{i}\right) \mathrm{d} \tau} \mathrm{d} \xi\right)\right] \mathrm{d} \eta \mathrm{d} a
\end{aligned}
$$

By using corollary 2, inequality $\left.{ }^{\star}\right)$ and Fatou's lemma, we have

$$
\lim _{t \rightarrow+\infty} \lambda_{i}(t) \leq \mathrm{e}^{R_{c}} \mathfrak{R}_{0}^{i} \limsup _{t \rightarrow+\infty} \lambda_{i}(t) .
$$

Since $\mathrm{e}^{R_{c}} \Re_{0}^{i}<1, \Rightarrow \limsup \sup _{t \rightarrow+\infty} \lambda_{i}(t)=0 \Rightarrow$

$$
\left\{\begin{array}{l}
\lim _{t \rightarrow+\infty} i_{i}(t, a)=\lim _{t \rightarrow+\infty} j_{i}(t, a)=\lim _{t \rightarrow+\infty} l_{i}(t, a)=0 \\
\lim _{t \rightarrow+\infty} s_{i}(t, a)=s_{i}^{0}(a), \lim _{t \rightarrow+\infty} v_{i}(t, a)=\Lambda_{i}-s_{i}^{0}(a) .
\end{array}\right.
$$

Corollary 3. The disease-free equilibrium is globally asymptotically in: 
with the initial conditions:

$$
\bar{s}_{i}^{*}(0)=\Lambda_{i} ; \bar{i}_{1}^{*}(0)=\bar{l}_{1}^{*}(0)=\bar{v}_{i}^{*}(0)=\bar{j}_{1}^{*}(0)=0 .
$$

Let

$$
h_{1}\left(\eta, \bar{\Gamma}_{1}^{*}\right)=\left(1-\phi_{1}\right) \bar{s}_{1}^{*}(\eta)+\delta_{1} \bar{v}_{1}^{*}(\eta)+\sigma_{1} \bar{j}_{1}^{*}(\eta) .
$$

Integrating system (31), we obtain: 


$$
\begin{aligned}
& \bar{S}_{1}^{*}(a)=\Lambda_{1} \mathrm{e}^{-\int_{0}^{a}\left[\bar{\Gamma}_{1}^{*} \beta_{1}(\tau) c_{1}(\tau) g_{1}(\tau)-\mu_{1}(\tau) \overrightarrow{\hat{i}_{1}^{*}}(\eta)+b(\eta)+\psi_{1}(\eta)\right] \mathrm{d} \eta} \\
& +\int_{0}^{a} b(\eta) \mathrm{e}^{-\int_{\eta}^{a}\left[\bar{\Gamma}_{1}^{*}\left(1-\phi_{1}\right) \beta_{1}(\tau) c_{1}(\tau) g_{1}(\tau)-\mu_{1}(\tau) \overrightarrow{\mathrm{i}}_{1}^{*}(\eta)+b(\eta)+\psi_{1}(\eta)\right] \mathrm{d} \eta} \mathrm{d} \eta \\
& \bar{i}_{1}^{*}(a)=\int_{0}^{a}\left[k_{1} \bar{l}_{1}^{*}(\eta)+\phi_{1} \beta_{1}(\eta) c_{1}(\eta) g_{1}(\eta) \bar{\Gamma}_{1}^{*} \bar{S}_{1}^{*}(\eta)\right] \mathrm{e}^{\left.-\int_{\eta}^{a}\left(r_{1}-\mu_{1}(\eta)\right)_{1}^{*}(\tau)+b(\tau)+\mu_{1}(\tau)\right) \mathrm{d} \tau} \mathrm{d} \eta \\
& \bar{j}_{1}^{*}(a)=\int_{0}^{a} \bar{i}_{1}^{*}(\eta) \mathrm{e}^{-\int_{\eta}^{a}\left(\sigma_{1} \beta_{1}(\tau) c_{1}(\tau) g_{1}(\tau) \bar{\Gamma}_{1}^{*}+b(\tau)-\mu_{1}(\tau) \bar{i}_{1}^{*}(\tau)\right) \mathrm{d} \tau} \mathrm{d} \eta \\
& \bar{v}_{1}^{*}(a)=\int_{0}^{a}\left[\psi_{1}(\eta) \bar{S}_{1}^{*}(\eta)+\rho_{2} \bar{V}_{2}^{*}(\eta)\right] \mathrm{e}^{-\int_{\eta}^{a}\left(\mathcal{S}_{1} \vec{\Gamma}_{1}^{*} c_{1}(\tau) g_{1}(\tau) \beta_{1}(\tau) \vec{\Gamma}_{1}^{*}+b(\tau)-\mu_{1}(\tau) \overrightarrow{\mathrm{i}}_{1}^{*}(\tau)\right) \mathrm{d} \tau} \\
& \bar{l}_{1}^{*}(a)=\bar{\Gamma}_{1}^{*} \int_{0}^{a} \mathrm{e}^{-\int_{\eta}^{a}\left(b(\tau)-\mu_{1}(\tau) \overrightarrow{\mathrm{l}}_{\mathrm{1}}^{*}(\tau)+k\right) \mathrm{d} \tau} \beta_{1}(\eta) c_{1}(\eta) g_{1}(\eta) \overline{\mathrm{s}}_{1}^{*}(\eta) h_{1}\left(\eta, \bar{\Gamma}_{1}^{*}\right) \mathrm{d} \eta \\
& \bar{S}_{2}^{*}(a)=\Lambda_{2} \mathrm{e}^{-\int_{0}^{a}\left(\psi_{2}(\tau)+b(\tau)-\mu_{1} \overrightarrow{1}^{*}(\tau)\right) \mathrm{d} \tau}+\int_{0}^{a} b(\eta) \mathrm{e}^{-\int_{\eta}^{a}\left(\psi_{2}(\tau)-\mu_{1}(\tau) \overrightarrow{1}_{1}^{*}(\tau)\right) \mathrm{d} \tau} \mathrm{d} \eta \\
& \bar{v}_{2}^{*}(a)=\int_{0}^{a}\left[\psi_{2}(\eta) \bar{s}_{2}^{*}(\eta)+\rho_{1} \bar{v}_{1}^{*}(\eta)\right] \mathrm{e}^{-\int_{\eta}^{a}\left(b(\tau)-\mu_{1}(\tau) \overline{\bar{i}}_{1}^{*}(\tau)\right) \mathrm{d} \tau} \mathrm{d} \eta .
\end{aligned}
$$

By injecting (37) in (34), we obtain:

$$
\begin{aligned}
{\overline{i_{1}}}^{*}(a)= & \bar{\Gamma}_{1}^{*} \int_{0}^{a} \beta_{1}(\eta) c_{1}(\eta) g_{1}(\eta) \mathrm{e}^{-\int_{\eta}^{a}\left(\beta(\tau)-\mu_{1}(\tau) \vec{i}_{1}^{*}(\tau)+r_{1}+\mu_{1}(\tau)\right) \mathrm{d} \tau} \\
& \times\left[\phi_{1} \overline{\mathrm{s}}_{1}^{*}(\eta)+k_{1} h_{1}\left(\eta, \bar{\Gamma}_{1}^{*}\right) \int_{\eta}^{a} \mathrm{e}^{-\int_{\xi}^{\eta}\left(r_{1}+\mu_{1}(\tau)-k_{1}\right) \mathrm{d} \tau} \mathrm{d} \xi\right] \mathrm{d} \eta
\end{aligned}
$$

Injecting (40) in the expression of $\bar{\Gamma}_{1}^{*}$, and dividing by $\bar{\Gamma}_{1}^{*}$ (since $\left.\bar{\Gamma}_{1}^{*} \neq 0\right)$ we obtain:

$$
\begin{aligned}
1= & \int_{0}^{a_{+}} \hat{\beta}_{1}(a) \int_{0}^{a} \beta_{1}(\eta) c_{1}(\eta) g_{1}(\eta) \mathrm{e}^{-\int_{\eta}^{a}\left(\beta(\tau)-\mu_{1}(\tau) \overline{\mathrm{r}}_{1}^{*}(\tau)+r_{1}+\mu_{1}(\tau)\right) \mathrm{d} \tau} \\
& \times\left[\phi_{1} \overline{\mathrm{s}}_{1}^{*}(\eta)+k_{1} h_{1}\left(\eta, \bar{\Gamma}_{1}^{*}\right) \int_{\eta}^{a} \mathrm{e}^{-\int_{\xi}^{\eta}\left(r_{1}+\mu_{1}(\tau)-k_{1}\right) \mathrm{d} \tau} \mathrm{d} \xi\right] \mathrm{d} \eta \mathrm{d} a
\end{aligned}
$$

Let $H_{1}$, the function define by:

$$
\begin{aligned}
H_{1}\left(\bar{\Gamma}_{1}^{*}\right)= & \int_{0}^{a_{+}} \hat{\beta}_{1}(a) \int_{0}^{a} \beta_{1}(\eta) c_{1}(\eta) g_{1}(\eta) e^{-\int_{\eta}^{a}\left(\beta(\tau)-\mu_{1}(\tau) \overrightarrow{\bar{\eta}}_{1}^{*}(\tau)+r_{1}+\mu_{1}(\tau)\right) \mathrm{d} \tau} \\
& \times\left[\phi_{1} \overline{\mathrm{s}}_{1}^{*}(\eta)+k_{1} h_{1}\left(\eta, \bar{\Gamma}_{1}^{*}\right) \int_{\eta}^{a} \mathrm{e}^{-\int_{\bar{\xi}}^{\eta}\left(r_{1}+\mu_{1}(\tau)-k_{1}\right) \mathrm{d} \tau} \mathrm{d} \xi\right] \mathrm{d} \eta \mathrm{d} a
\end{aligned}
$$

Since $h_{1}(\eta, 0)=N_{\psi_{1}}(\eta)$ i.e. when $\bar{\Gamma}_{1}^{*}=0, \bar{s}_{i}^{*}(a)=s_{i}^{0}(a)$ and $\bar{v}_{i}^{*}(a)=v_{i}^{0}(a)$, so the net reproductive number is given by

$$
H_{1}(0)=\mathfrak{R}^{1}\left(\psi_{1}\right)
$$

i.e.

$$
\mathfrak{R}^{1}\left(\psi_{1}\right)=\int_{0}^{a_{+}} \hat{\beta}_{1}(a) \int_{0}^{a} \mathrm{e}^{-\int_{\eta}^{a}\left(b(\tau)+\mu_{1}(\tau)+r_{1}\right) \mathrm{d} \tau} \beta_{1}(\eta) c_{1}(\eta) g_{1}(\eta)\left[\phi_{1} s_{1}^{0}(\eta)+k_{1} N_{\psi_{1}}(\eta) \int_{\eta}^{a} \mathrm{e}^{-\int_{\alpha}^{\eta}\left(\mu_{1}(\tau)+r_{1}-k_{1}\right) \mathrm{d} \tau} \mathrm{d} \alpha\right] \mathrm{d} \eta \mathrm{d} a .
$$

We now see that an endemic steady state exists if Equation (41) has a positive solution.

Since $H_{1}(0)=\Re_{1}\left(\psi_{1}\right)$, hence $H_{1}(0)>1$. We know that

$$
\bar{s}_{1}^{*}(a)+\bar{l}_{1}^{*}(a)+\bar{i}_{1}^{*}(a)+\bar{v}_{1}^{*}(a)+\bar{j}_{1}^{*}(a)=\Lambda_{1}<1 \text {. Hence }
$$

$$
\bar{i}_{1}^{*}(a)<1 \text {. }
$$


Since $\bar{\Gamma}_{1}^{*}>0$, from (42) and (43) we obtain:

$$
\begin{aligned}
\bar{\Gamma}_{1}^{*} H_{1}\left(\bar{\Gamma}_{1}^{*}\right)= & \int_{0}^{a_{+}} \hat{\beta}_{1}(a) \int_{0}^{a} \bar{\Gamma}_{1}^{*} \beta_{1}(\eta) c_{1}(\eta) g_{1}(\eta) \mathrm{e}^{-\int_{\eta}^{a}\left(\beta(\tau)-\mu_{1}(\tau) \bar{i}_{1}^{*}(\tau)+r_{1}+\mu_{1}(\tau)\right) \mathrm{d} \tau} \\
& \times\left[\phi_{1} \bar{S}_{1}^{*}(\eta)+k_{1} h_{1}\left(\eta, \bar{\Gamma}_{1}^{*}\right) \int_{\eta}^{a} \mathrm{e}^{-\int_{\xi}^{\eta}\left(r_{1}+\mu_{1}(\tau)-k_{1}\right) \mathrm{d} \tau} \mathrm{d} \xi\right] \mathrm{d} \eta \mathrm{d} a \\
& <\int_{0}^{a_{+}} \hat{\beta}_{1}(a) \mathrm{d} a=\beta_{1}^{+} .
\end{aligned}
$$

In particular, for $\bar{\Gamma}_{1}^{*}=\beta_{1}^{+}$, we have $H\left(\beta_{1}^{+}\right)<1$, but $H(0)>1$. Since $H_{1}$ is continous function of $\bar{\Gamma}_{1}^{*}$, we conclude that $H\left(\bar{\Gamma}_{1}^{*}\right)=1$, has a positive solution $\hat{\Gamma}_{1}^{*}$ on $0 ; \beta^{+}\left[\right.$. This solution may not be unique since $H$ may not be monotone $\left(H\left(\bar{\Gamma}_{1}^{*}\right)\right.$ depends on $h_{1}\left(\eta, \bar{\Gamma}_{1}^{*}\right)$ which is defined implicitly). It follows that when $\mathfrak{R}^{1}\left(\psi_{1}\right)>1$, there exists an endemic steady state distribution which is given by the unique solution of Equation (41) corresponding to $\hat{\Gamma}_{1}^{*}$.

\subsection{The Second Boundary Endemic Equilibrium}

Theorem 5. A boundary endemic equilibrium of the form

$\bar{E}_{2}^{* * *}=\left(\bar{s}_{1}^{* *}(a), 0,0, \bar{v}_{1}^{* *}(a), \bar{s}_{2}^{* *}(a), \bar{l}_{2}^{* *}(a), \bar{i}_{2}^{* *}(a), \bar{j}_{2}^{* *}(a), \bar{v}_{2}^{* *}(a)\right)$ whenever

$\mathfrak{R}^{1}\left(\psi_{1}\right)<1$ and $\mathfrak{R}^{2}\left(\psi_{2}\right)>1$. This means that the disease is dies out in the first subpopulation and is endemic in the second sub-population.

Proof. (Ideas of proof)

$\bar{E}_{2}^{* *}=\left(\bar{s}_{1}^{* *}(a), 0,0, \bar{v}_{1}^{* *}(a), \bar{s}_{2}^{* *}(a), \bar{l}_{2}^{* *}(a), \bar{i}_{2}^{* *}(a), \bar{j}_{2}^{* *}(a), \bar{v}_{2}^{* *}(a)\right)$ satisfies the following equations:

$$
\left\{\begin{array}{l}
\frac{\mathrm{d}}{\mathrm{d} a} \bar{s}_{1}^{* *}(a)=b_{1}(a)-\left[\psi_{1}(a)+b(a)-\mu_{2}(a) \bar{i}_{2}^{* *}(a)\right] \bar{s}_{1}^{* *}(a) \\
\frac{\mathrm{d}}{\mathrm{d} a} \bar{v}_{1}^{* *}(a)=\psi_{1}(a) \bar{s}_{1}^{* *}(a)+\rho_{2} \bar{v}_{2}^{* *}(a)-\left(b(a)-\mu_{2}(a) \bar{i}_{2}^{* *}(a)\right) \bar{v}_{1}^{* *}(a) \\
\frac{\mathrm{d}}{\mathrm{d} a} \bar{s}_{2}^{* *}(a)=b_{2}(a)-\left[\beta_{2}(a) c_{2}(a) g_{2}(a) \bar{\Gamma}_{2}^{* *}+\psi_{2}(a)+b(a)-\mu_{2}(a) \bar{i}_{2}^{* *}(a)\right] s_{2}^{* *}(a) \\
\frac{\mathrm{d}}{\mathrm{d} a} \bar{l}_{2}^{* *}(a)=\beta_{2}(a) c_{2}(a) g_{2}(a) \bar{\Gamma}_{2}^{* *}\left[\left(1-\phi_{2}\right) \bar{s}_{2}^{* *}(a)+\delta_{2} \bar{v}_{2}^{* *}(a)+\sigma_{2} \bar{j}_{2}^{* *}(a)\right]-\left(b(a)+k_{2}-\mu_{2}(a) \bar{i}_{2}^{* *}(a)\right) \bar{l}_{2}^{* *}(a) \\
\frac{\mathrm{d}}{\mathrm{d} a} \bar{i}_{2}^{* *}(a)=k_{2} \bar{l}_{2}^{* *}(a)+\phi_{2} \beta_{2}(a) c_{2}(a) g_{2}(a) \bar{\Gamma}_{2}^{*} \bar{s}_{2}^{*}(a)-\left[r_{2}+b(a)+\mu_{2}(a)-\mu_{2}(a) \bar{i}_{2}^{* *}(a)\right] \bar{i}_{2}^{* *}(a) \\
\frac{\mathrm{d}}{\mathrm{d} a} \bar{j}_{2}^{* *}(a)=r_{2} \bar{i}_{2}^{* *}(a)-\left(\sigma_{2} \beta_{2}(a) c_{2}(a) g_{2}(a) \bar{\Gamma}_{1}^{* *}+b(a)-\mu_{2}(a) \bar{i}_{2}^{* *}(a)\right) \bar{j}_{2}^{* *}(a) \\
\frac{\mathrm{d}}{\mathrm{d} a} \bar{v}_{2}^{* *}(a)=\psi_{2}(a) \bar{s}_{2}^{* *}(a)+\rho_{2} \bar{v}_{2}^{* *}(a)-\left(\delta_{2} \beta_{2}(a) c_{2}(a) g_{2}(a) \bar{\Gamma}_{2}^{* *}+b(a)-\mu_{2}(a) \bar{i}_{2}^{* *}(a)\right) v_{2}^{* *}(a) \\
\bar{\Gamma}_{2}^{* *}=\int_{0}^{a_{+}} \hat{\beta}_{2}(a) \bar{i}_{2}^{* *}(a) \mathrm{d} a
\end{array}\right.
$$

with the initial conditions:

$$
\bar{s}_{i}^{* *}(0)=\Lambda_{i} ; \bar{i}_{2}^{* *}(0)=\bar{l}_{2}^{* *}(0)=\bar{v}_{i}^{* *}(0)=\bar{j}_{2}^{* *}(0)=0 .
$$

Let

$$
h_{2}\left(\eta, \bar{\Gamma}_{2}^{* *}\right)=\left(1-\phi_{2}\right) \bar{s}_{2}^{* *}(\eta)+\delta_{2} \bar{v}_{2}^{* *}(\eta)+\sigma_{2} \bar{j}_{2}^{* *}(\eta) .
$$

Integrating system (51), we obtain:

$$
\bar{S}_{1}^{* *}(a)=\Lambda_{1} \mathrm{e}^{-\int_{0}^{a}\left(\psi_{1}(\tau)+b(\tau)-\mu_{2} \bar{i}_{2}^{*}(\tau)\right) \mathrm{d} \tau}+\int_{0}^{a} b(\eta) \mathrm{e}^{-\int_{\eta}^{a}\left(\psi_{1}(\tau)-\mu_{2}(\tau) \bar{i}_{2}^{* *}(\tau)\right) \mathrm{d} \tau} \mathrm{d} \eta
$$




$$
\begin{aligned}
& \bar{V}_{1}^{* *}(a)=\int_{0}^{a}\left[\psi_{2}(\eta) \bar{S}_{2}^{* *}(\eta)+\rho_{1} \bar{v}_{1}^{* *}(\eta)\right] \mathrm{e}^{-\int_{\eta}^{a}\left(b(\tau)-\mu_{1}(\tau) \overrightarrow{1}_{1}^{* *}(\tau)\right) \mathrm{d} \tau} \mathrm{d} \eta \\
& \bar{S}_{2}^{* *}(a)=\Lambda_{2} \mathrm{e}^{-\int_{0}^{a}\left[\bar{\Gamma}_{2}^{*} \beta_{2}(\tau) c_{2}(\tau) g_{2}(\tau)-\mu_{2}(\tau) \overrightarrow{i_{2} *}(\eta)+b(\eta)+\psi_{2}(\eta)\right] \mathrm{d} \eta} \\
& +\int_{0}^{a} b(\eta) \mathrm{e}^{-\int_{\eta}^{a}\left[\bar{\Gamma}_{2}^{* *}\left(1-\phi_{2}\right) \beta_{2}(\tau) c_{2}(\tau) g_{2}(\tau)-\mu_{2}(\tau) \bar{i}_{2}^{* *}(\tau)+b(\tau)+\psi_{2}(\tau)\right] \mathrm{d} \tau} \mathrm{d} \eta \\
& \bar{i}_{2}^{* *}(a)=\int_{0}^{a}\left[k_{2} \bar{l}_{2}^{* *}(\eta)+\phi_{2} \beta_{2}(\eta) c_{2}(\eta) g_{2}(\eta) \bar{\Gamma}_{2}^{*} \bar{S}_{2}^{* *}(\eta)\right] \mathrm{e}^{-\iint_{\eta}^{a}\left(r_{1}-\mu_{1}(\eta) \bar{i}_{2}^{* *}(\tau)+b(\tau)+\mu_{2}(\tau)\right) \mathrm{d} \tau} \mathrm{d} \eta \\
& \bar{j}_{2}^{* *}(a)=\int_{0}^{a} \bar{i}_{2}^{* *}(\eta) \mathrm{e}^{-\int_{\eta}^{a}\left(\sigma_{2} \beta_{2}(\tau) c_{2}(\tau) g_{2}(\tau) \bar{\Gamma}_{2}^{* *}+b(\tau)-\mu_{2}(\tau) \overline{\bar{i}}_{2}^{* *}(\tau)\right) \mathrm{d} \tau} \mathrm{d} \eta \\
& \bar{V}_{2}^{* *}(a)=\int_{0}^{a}\left[\psi_{2}(\eta) \bar{S}_{2}^{* *}(\eta)+\rho_{1} \bar{V}_{1}^{* *}(\eta)\right] \mathrm{e}^{-\int_{\eta}^{a}\left(\delta_{2} \bar{\Gamma}_{2}^{* *} c_{2}(\tau) g_{2}(\tau) \beta_{2}(\tau) \bar{\Gamma}_{2}^{* *}+b(\tau)-\mu_{2}(\tau) \bar{l}_{2}^{* *}(\tau)\right) \mathrm{d} \tau} \\
& \bar{l}_{2}^{* *}(a)=\bar{\Gamma}_{2}^{*} \int_{0}^{a} \mathrm{e}^{-\int_{\eta}^{a}\left(b(\tau)-\mu_{2}(\tau) \bar{i}_{2}^{* *}(\tau)+k\right) \mathrm{d} \tau} \beta_{2}(\eta) c_{2}(\eta) g_{2}(\eta) \bar{s}_{2}^{* *}(\eta) h_{2}\left(\eta, \bar{\Gamma}_{2}^{*}\right) \mathrm{d} \eta .
\end{aligned}
$$

Hence, by the similar method using in theorem 4, we obtain the result.

\subsection{The Interior Endemic Equilibrium}

\section{Theorem 6. An interior endemic equilibrium of the form}

$$
E^{*}=\left(s_{1}^{*}(a), l_{1}^{*}(a), i_{1}^{*}(a), j_{1}^{*}(a), v_{1}^{*}(a), s_{2}^{*}(a), l_{2}^{*}(a), i_{2}^{*}(a), j_{2}^{*}(a), v_{2}^{*}(a)\right)
$$

whenever $\mathfrak{R}^{1}\left(\psi_{1}\right)>1$ and $\mathfrak{R}^{2}\left(\psi_{2}\right)>1$, which corresponds to case when the disease persists in the two sub-populations.

Proof. $E^{*}=\left(s_{1}^{*}(a), l_{1}^{*}(a), i_{1}^{*}(a), j_{1}^{*}(a), v_{1}^{*}(a), s_{2}^{*}(a), l_{2}^{*}(a), i_{2}^{*}(a), j_{2}^{*}(a), v_{2}^{*}(a)\right)$ satisfies the following equations:

$$
\left\{\begin{array}{l}
\frac{\mathrm{d}}{\mathrm{d} a} s_{1}^{*}(a)=b_{1}(a)-\left[\beta_{1}(a) c_{1}(a) g_{1}(a) \Gamma_{1}^{*}+\psi_{1}(a)+b(a)-\mu_{1}(a) i_{1}^{*}(a)-\mu_{2}(a) i_{2}^{*}(a)\right] s_{1}^{*}(a) \\
\frac{\mathrm{d}}{\mathrm{d} a} l_{1}^{*}(a)=\beta_{1}(a) c_{1}(a) g_{1}(a) \Gamma_{1}^{*}\left[\left(1-\phi_{1}\right) s_{1}^{*}(a)+\delta_{1} v_{1}^{*}(a)+\sigma_{1} j_{1}^{*}(a)\right]-\left(b(a)+k_{1}-\mu_{1}(a) i_{1}^{*}(a)-\mu_{2}(a) i_{2}^{*}(a)\right) l_{1}^{*}(a) \\
\frac{\mathrm{d}}{\mathrm{d} a} i_{1}^{*}(a)=k_{1} l_{1}^{*}(a)+\phi_{1} \beta_{1}(a) c_{1}(a) g_{1}(a) \Gamma_{1}^{*} s_{1}^{*}(a)-\left[r_{1}+b(a)+\mu_{1}(a)-\mu_{1}(a) i_{1}^{*}(a)-\mu_{2}(a) i_{2}^{*}(a)\right] i_{1}^{*}(a) \\
\frac{\mathrm{d}}{\mathrm{d} a} j_{1}^{*}(a)=r_{1} i_{1}^{*}(a)-\left(\sigma_{1} \beta_{1}(a) c_{1}(a) g_{1}(a) \Gamma_{1}^{*}+b(a)-\mu_{1}(a) i_{1}^{*}(a)-\mu_{2}(a) i_{2}^{*}(a)\right) j_{1}^{*}(a) \\
\frac{\mathrm{d}}{\mathrm{d} a} v_{1}^{*}(a)=\psi_{1}(a) s_{1}^{*}(a)+\rho_{2} v_{2}^{*}(a)-\left(\delta_{1} \beta_{1}(a) c_{1}(a) g_{1}(a) \Gamma_{1}^{*}+b(a)-\mu_{1}(a) i_{1}^{*}(a)-\mu_{2}(a) i_{2}^{*}(a)+\rho_{1}\right) v_{1}^{*}(a) \\
\frac{\mathrm{d}}{\mathrm{d} a} s_{2}^{*}(a)=b_{2}(a)-\left[\beta_{2}(a) c_{2}(a) g_{2}(a) \Gamma_{2}+\psi_{2}(a)+b(a)-\mu_{1}(a) i_{1}^{*}(a)-\mu_{2}(a) i_{2}(a)\right] s_{2}(a) \\
\frac{\mathrm{d}}{\mathrm{d} a} l_{2}^{*}(a)=\beta_{2}(a) c_{2}(a) g_{2}(a) \Gamma_{2}^{*}\left[\left(1-\phi_{2}\right) s_{2}^{*}(a)+\delta_{2} v_{2}^{*}(a)+\sigma_{2} j_{2}^{*}(a)\right]-\left(b(a)+k_{2}-\mu_{1}(a) i_{1}^{*}(a)-\mu_{2}(a) i_{2}^{*}(a)\right) l_{2}^{*}(a) \\
\frac{\mathrm{d}}{\mathrm{d} a} i_{2}^{*}(a)=k_{2} l_{2}^{*}(a)+\phi_{2} \beta_{2}(a) c_{2}(a) g_{2}(a) \Gamma_{2}^{*} s_{2}^{*}(a)-\left[r_{2}+b(a)+\mu_{2}(a)-\mu_{1}(a) i_{1}^{*}(a)-\mu_{2}(a) i_{2}^{*}(a)\right] i_{2}^{*}(a) \\
\frac{\mathrm{d}}{\mathrm{d} a} j_{2}(a)=r_{2} i_{2}^{*}(a)-\left(\sigma_{2} \beta_{2}(a) c_{2}(a) g_{2}(a) \Gamma_{2}^{*}+b(a)-\mu_{1}(a) i_{1}^{*}(a)-\mu_{2}(a) i_{2}^{*}(a)\right) j_{2}^{*}(a) \\
\frac{\mathrm{d}}{\mathrm{d} a} v_{2}^{*}(a)=\psi_{2}(a) s_{2}^{*}(a)+\rho_{1} v_{1}^{*}(a)-\left(\delta_{2} \beta_{2}(a) c_{2}(a) g_{2}(a) \Gamma_{2}^{*}+b(a)-\mu_{1}(a) i_{1}^{*}(a)-\mu_{2}(a) i_{2}^{*}(a)+\rho_{2}\right) v_{2}^{*}(a) \\
\Gamma_{i}^{*}=\int_{0}^{a_{+}} \hat{\beta}_{i}(a) i_{i}^{*}(a) \mathrm{d} a
\end{array}\right.
$$


with the initial conditions:

$$
\begin{gathered}
S_{i}^{*}(0)=\Lambda_{i} ; \quad l_{i}^{*}(0)=i_{i}^{*}(0)=j_{i}^{*}(0)=v_{i}^{*}(0)=0 \\
S_{i}^{*}(a)=\Lambda_{i} \mathrm{e}^{-\int_{0}^{a}\left[\beta_{i}(\tau) c_{i}(\tau) g_{i}(\tau) \Gamma^{*}+b(\tau)-\mu_{1}(\tau) i_{1}^{*}(\tau)-\mu_{2}(\tau) i_{2}^{*}(\tau)\right] \mathrm{d} \tau} \\
+\int_{0}^{a} b_{i}(\eta) \mathrm{e}^{-\int_{\eta}^{a}\left[\beta_{i}(\tau) c_{i}(\tau) g_{i}(\tau) \Gamma^{*}+b(\tau)-\mu_{1}(\tau) i_{1}^{*}(\tau)-\mu_{2}(\tau) i_{2}^{*}(\tau)\right] \mathrm{d} \tau} \mathrm{d} \eta .
\end{gathered}
$$

Let

$$
\begin{gathered}
h_{i}^{*}\left(\eta, \Gamma_{i}^{*}\right)=\left(1-\phi_{i}\right) s_{i}^{*}(\eta)+\delta_{i} v_{i}^{*}(\eta)+\sigma_{i} j_{i}^{*}(\eta) \\
l_{i}^{*}(a)=\Gamma_{i}^{*} \int_{0}^{a} \beta_{i}(\eta) c_{i}(\eta) g_{i}(\eta) h_{i}\left(\eta, \Gamma_{i}^{*}\right) \mathrm{e}^{-\int_{\eta}^{a}\left(b(\tau)-\mu_{1}(\tau) i_{i}^{*}(\tau)-\mu_{2}(\tau) i_{2}^{*}(\tau)\right) \mathrm{d} \tau} \mathrm{d} \eta \\
i_{i}^{*}(a)=\int_{0}^{a}\left[k_{i} l_{i}^{*}(\eta)+\phi_{i} \beta_{i}(\eta) c_{i}(\eta) g_{i}(\eta) \Gamma_{i}^{*} s_{i}^{*}(\eta)\right] \mathrm{e}^{-\int_{\eta}^{a}\left(b(\tau)+r_{i}+\mu_{i}(\tau)-\mu_{1}(\tau) i_{1}^{*}(\tau)-\mu_{2}(\tau) i_{2}(\tau)\right) \mathrm{d} \tau} \mathrm{d} \eta \\
j_{i}^{*}(a)=r_{i} \int_{0}^{a} \dot{l}_{i}^{*}(\eta) \mathrm{e}^{-\int_{\eta}^{a}\left(\sigma_{i} \beta_{i}(\tau) c_{i}(\tau) g_{i}(\tau) \Gamma_{i}^{*}+b(\tau)-\mu_{1}(\tau) i_{1}^{*}(\tau)-\mu_{2}(\tau) i_{2}^{*}(\eta)\right) \mathrm{d} \tau} \mathrm{d} \eta \\
v_{2}^{*}(a)=\int_{0}^{a}\left(\psi_{1}(\eta) s_{1}^{*}(\eta)+\rho_{2} v_{2}^{*}(\eta)\right) \mathrm{e}^{-\int_{\eta}^{a}\left(\delta_{1} \beta_{1}(\tau) c_{1}(\tau) g_{1}(\tau) \Gamma_{1}^{*}+b(\tau)-\mu_{1}(\tau) i_{1}^{*}(\tau)-\mu_{2}(\tau) i_{2}^{*}(\tau)\right) \mathrm{d} \tau} \mathrm{d} \eta \\
v_{0}^{a}\left(\psi_{2}(\eta) s^{*}(\eta)+\rho_{1} v_{1}^{*}(\eta)\right) \mathrm{e}^{-\int_{\eta}^{a}\left(\delta_{2} \beta_{2}(\tau) c_{2}(\tau) g_{2}(\tau) \Gamma^{*}+b(\tau)-\mu_{1}(\tau) i_{1}^{*}(\tau)-\mu_{2}(\tau) i_{2}^{*}(\tau)\right) \mathrm{d} \tau} \mathrm{d} \eta .
\end{gathered}
$$

By injecting (58) in (59), we obtain:

$$
\begin{aligned}
i_{i}^{*}(a)= & \Gamma_{i}^{*} \int_{0}^{a} \beta_{i}(\eta) c_{i}(\eta) g_{i}(\eta) \mathrm{e}^{-\int_{\eta}^{a}\left(b(\tau)-\mu_{1}(\tau) i_{1}^{*}(\tau)-\mu_{2}(\tau) i_{2}^{*}(\tau)-r_{i}+\mu_{i}(\tau)\right) \mathrm{d} \tau} \\
& \times\left[\phi_{i} s_{i}^{*}(\eta)+k_{i} h_{i}\left(\eta, \Gamma_{i}^{*}\right) \int_{\eta}^{a} \mathrm{e}^{-\int_{\xi}^{\eta}\left(r_{i}+\mu_{i}(\tau)-k_{i}\right) \mathrm{d} \tau} \mathrm{d} \xi\right] \mathrm{d} \eta .
\end{aligned}
$$

By injecting (63) in the expression of $\Gamma_{i}^{*}$, and dividing by $\Gamma_{i}^{*}\left(\right.$ since $\left.\Gamma_{i}^{*} \neq 0\right)$ we obtain:

$$
\begin{aligned}
1= & \int_{0}^{a_{+}} \hat{\beta}_{i}(a) \int_{0}^{a} \beta_{i}(\eta) c_{i}(\eta) g_{i}(\eta) \mathrm{e}^{-\int_{\eta}^{a}\left(b(\tau)-\mu_{1}(\tau) i_{1}^{*}(\tau)-\mu_{2}(\tau) i_{2}^{*}(\tau)-r_{i}+\mu_{i}(\tau)\right) \mathrm{d} \tau} \\
& \times\left[\phi_{i} s_{i}^{*}(\eta)+k_{i} h_{i}\left(\eta, \Gamma_{i}^{*}\right) \int_{\eta}^{a} \mathrm{e}^{-\int_{\xi}^{\eta}\left(r_{i}+\mu_{i}(\tau)-k_{i}\right) \mathrm{d} \tau} \mathrm{d} \xi\right] \mathrm{d} \eta \mathrm{d} a .
\end{aligned}
$$

Let $H_{i}$, the function define by:

$$
\begin{aligned}
H_{i}\left(\Gamma_{i}^{*}\right)= & \int_{0}^{a_{+}} \hat{\beta}_{i}(a) \int_{0}^{a} \beta_{i}(\eta) c_{i}(\eta) g_{i}(\eta) \mathrm{e}^{-\int_{\eta}^{a}\left(b(\tau)-\mu_{1}(\tau) i_{1}^{*}(\tau)-\mu_{2}(\tau) i_{2}^{*}(\tau)-r_{i}+\mu_{i}(\tau)\right) \mathrm{d} \tau} \\
& \times\left[\phi_{i} s_{i}^{*}(\eta)+k_{i} h_{i}\left(\eta, \Gamma_{i}^{*}\right) \int_{\eta}^{a} \mathrm{e}^{-\int_{\xi}^{\eta}\left(r_{i}+\mu_{i}(\tau)-k_{i}\right) \mathrm{d} \tau} \mathrm{d} \xi\right] \mathrm{d} \eta \mathrm{d} a .
\end{aligned}
$$

Since $h_{i}(\eta, 0)=N_{\psi_{i}}(\eta)$ i.e. when $\Gamma_{i}^{*}=0, s_{i}^{*}(a)=s_{i}^{0}(a)$ and $v_{i}^{*}(a)=v_{i}^{0}(a)$, so the net reproductive number is given by

$$
H_{i}(0)=\mathfrak{R}^{i}\left(\psi_{i}\right) \text {, i.e. }
$$

$$
\mathfrak{R}^{i}\left(\psi_{i}\right)=\int_{0}^{a_{+}} \hat{\beta}_{i}(a) \int_{0}^{a} \mathrm{e}^{-\int_{\eta}^{a}\left(b(\tau)+\mu_{i}(\tau)+r_{i}\right) \mathrm{d} \tau} \beta_{i}(\eta) c_{i}(\eta) g_{i}(\eta)\left[\phi_{i} s_{i}^{0}(\eta)+k_{i} N_{\psi_{i}}(\eta) \int_{\eta}^{a} \mathrm{e}^{-\int_{\alpha}^{\eta}\left(\mu_{i}(\tau)+r_{i}-k_{i}\right) \mathrm{d} \tau} \mathrm{d} \alpha\right] \mathrm{d} \eta \mathrm{d} a .
$$

We now see that an endemic steady state exists if Equation (64) has a positive solution. Since

$H_{i}(0)=\mathfrak{R}^{i}\left(\psi_{i}\right)$, hence $H_{i}(0)>1$. We know that 


$$
\begin{aligned}
s_{i}^{*}(a)+l_{i}^{*}(a)+i_{i}^{*}(a)+v_{i}^{*}(a)+j_{i}^{*}(a)= & \Lambda_{i}<1 . \text { Hence } \\
& i_{i}^{*}(a)<1
\end{aligned}
$$

Since $\Gamma_{i}^{*}>0$, from (65) and (66) we obtain:

$$
\begin{aligned}
\Gamma_{i}^{*} H_{i}\left(\Gamma_{i}^{*}\right)= & \int_{0}^{a_{+}} \hat{\beta}_{i}(a) \int_{0}^{a} \Gamma_{i}^{*} \beta_{i}(\eta) c_{i}(\eta) g_{i}(\eta) \mathrm{e}^{-\int_{\eta}^{a}\left(\beta(\tau)-\mu_{1}(\tau) i_{1}^{*}(\tau)-\mu_{2}(\tau) i_{i}^{*}(\tau)-r_{i}+\mu_{i}(\tau)\right) \mathrm{d} \tau} \\
& \times\left[\phi_{i} s_{i}^{*}(\eta)+k_{i} h_{i}\left(\eta, \Gamma_{i}^{*}\right) \int_{\eta}^{a} \mathrm{e}^{-\int_{\xi}^{\eta}\left(r_{i}+\mu_{i}(\tau)-k_{i}\right) \mathrm{d} \tau} \mathrm{d} \xi\right] \mathrm{d} \eta \mathrm{d} a \\
& <\int_{0}^{a_{+}} \hat{\beta}_{i}(a) \mathrm{d} a=\beta_{i}^{+} .
\end{aligned}
$$

In particular, for $\Gamma_{i}^{*}=\beta_{i}^{+}$, we have $H_{i}\left(\beta_{i}^{+}\right)<1$, but $H_{i}(0)>1$. Since $H_{i}$ is continous function of $\Gamma_{i}^{*}$, we conclude that $H\left(\Gamma_{i}^{*}\right)=1$, has a positive solution $\hat{\Gamma}_{i}^{*}$ on ] $0 ; \beta_{i}^{+}\left[\right.$. This solution may not be unique since $H_{i}$ may not be monotone $\left(H\left(\Gamma_{i}^{*}\right)\right.$ depends on $h\left(\eta, \Gamma_{i}^{*}\right)$ which is defined implicitly). It follows that when $\mathfrak{R}^{i}\left(\psi_{i}\right)>1$, there exists an endemic steady state distribution which is given by the unique solution of Equation (64) corresponding to $\hat{\Gamma}_{i}^{*}$.

\subsection{Simulation}

In this section, when $\mathfrak{R}^{1}\left(\psi_{1}\right)>1$ and $\mathfrak{R}^{2}\left(\psi_{2}\right)>1$ we will evaluate the impact of BCG vaccine and the birth rate of the population in the dynamics of spread of TB. Assuming that all parameters are the same in both patches except the vaccine rate, we observe an increase in the number of infected if the vaccination rate decreases (Figure 2). Also taking the same parameters except birth rates, we see an increased number of infected if the rate increases (Figure 3).

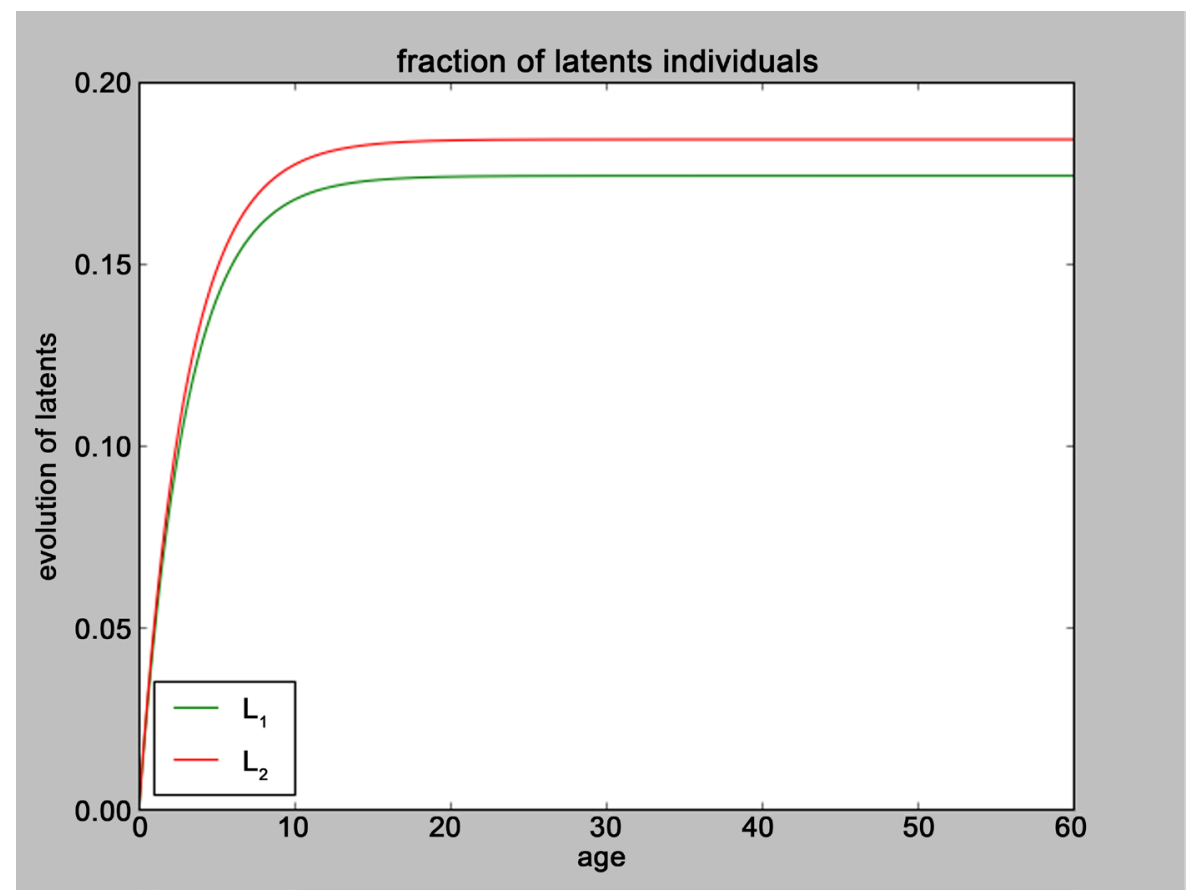

Figure 2. Evolution of the number of latents individuals with $\psi_{1}(a)=0.462$ and $\psi_{2}(a)=0.365$. 


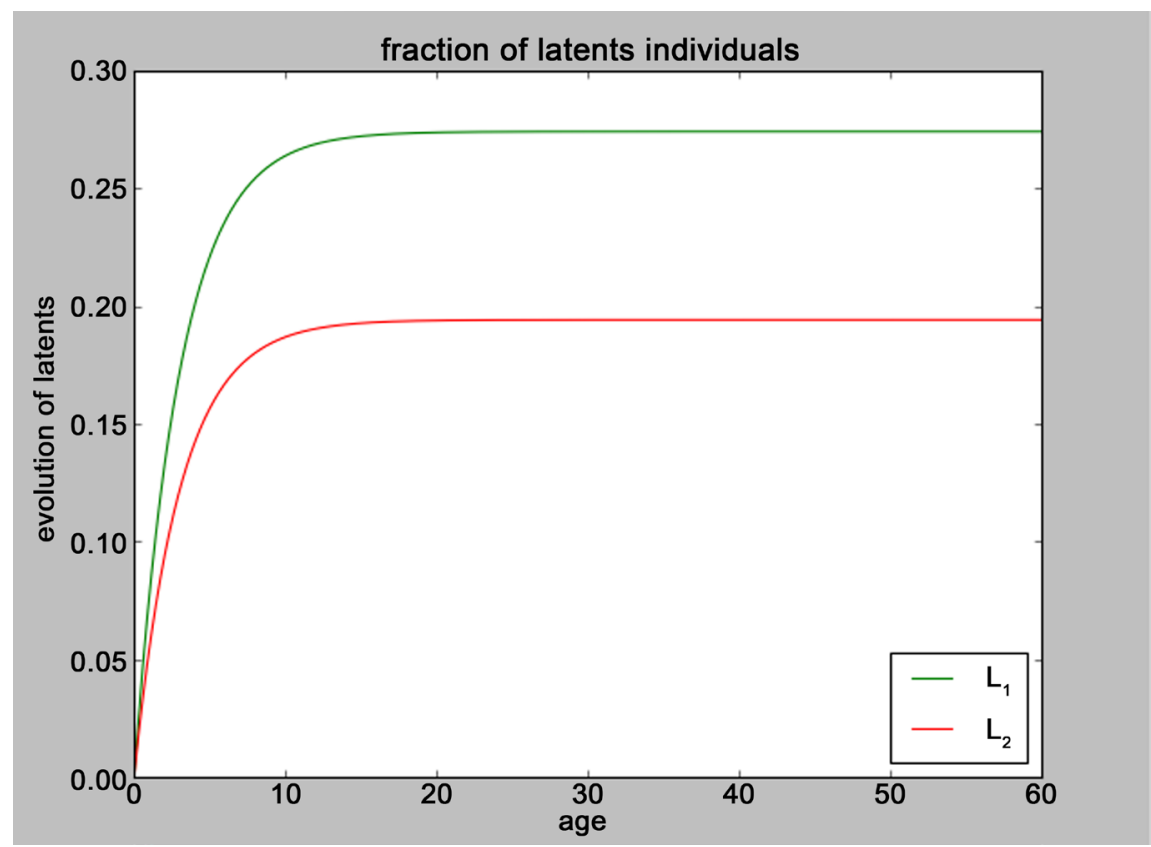

Figure 3. Evolution of the number of latents individuals with $b_{1}(a)=0.0461$ and $b_{2}(a)=0.0318$.

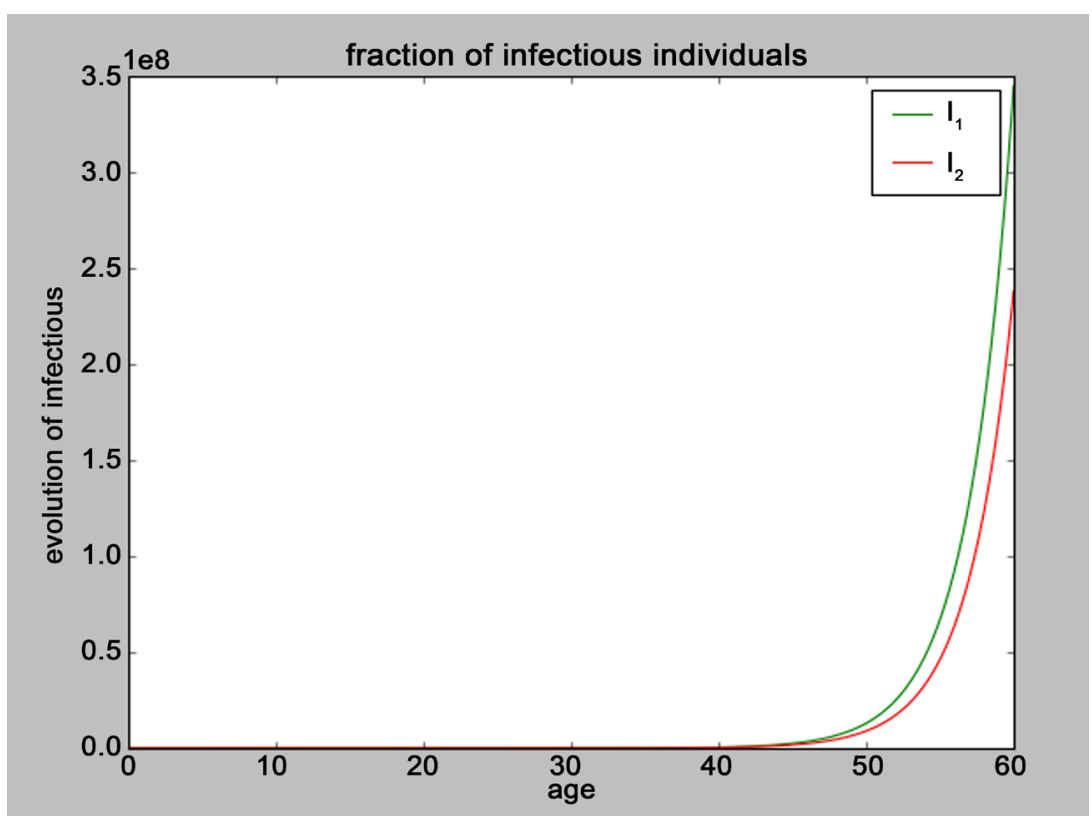

Figure 4. Evolution of the number of infectious individuals when: $\mathfrak{R}^{1}\left(\psi_{1}\right)=0.572$ and $\mathfrak{R}^{2}\left(\psi_{2}\right)=0.435 \quad\left(\psi_{2}=0.852\right.$ and $\left.\psi_{1}=0.785\right)$.

When $\mathfrak{R}^{1}\left(\psi_{1}\right)=0.572$ and $\mathfrak{R}^{2}\left(\psi_{2}\right)=0.435 \quad\left(\psi_{2}=0.852\right.$ and $\left.\psi_{1}=0.785\right)$, we have the evolution of the number of infectious individuals (Figure 4 ).

\section{Discussion, Conclusion and Future Work}

In this paper, an age structured model of two-patch for tuberculosis was analyzed and 
discussed. Each sub-population is subjected to a vaccination program. Apart from age; the vaccinated compartment, we introduced as a class of treated in the model proposed by Tewa J. Jules in [11] and allowed the migration of vaccinated population. The same result was found if the most susceptible migrated too. Although some studies have shown an ineffectiveness of BCG in the prevention of tuberculosis [21], our work demonstrated the contribution of BCG in the process of eradicating TB. The negative impact of the increase in the birth rate was shown. If we neglect the mortality death rate linked to the disease, we obtain the only usual condition of global stability to the disease free equilibrium i.e. $\mathfrak{R}_{0}<1$. It remains for us many challenges such as the endemic equilibrium points of this model and the one of [8] to deal with. For future work, in order to study the real impact of the tuberculosis migration in the dynamic of the expansion of the disease, we will use this model and authorize the migration of all individuals (i.e. susceptible, infected, infectious, vaccinated and treated).

\section{Acknowledgements}

We thank the Editor and the referee for their comments. We would like to thank $\mathrm{Nu}$ merical Analysis student group for their valuable comments and the authors whose works have been used in this article. We also thank the ministry of Higher Education of Research an Innovation who kindly supported the costs of the publication.

\section{References}

[1] Rohaeti, E., Wardatun, S. and Andriyati, A. (2015) Stability Analysis Model of Spreading and Controlling of Tuberculosis. Applied Mathematical Sciences, 9, 2559-2566. http://dx.doi.org/10.12988/ams.2015.52100

[2] Silva, C.J. and Torres, D.F.M. (2015) A TB-HIV/AIDS Coinfection Model and Optimal Control Treatment. Discrete and Continuous Dynamical Systems, 35, 4639-4663. http://dx.doi.org/10.3934/dcds.2015.35.4639

[3] Echeng, B.B. and Lebedev, K.A. (2015) On Mathematical Modeling of the Effect of BiTherapeutic Treatment of Tuberculosis Epidemic. Journal of Modern Mathematics and Statistics, 9, 1-7.

[4] Raoult, D. (2006) Epidémie et maladies infectieuse dans l'histoire.

[5] World Health Organization (2005) Global Tuberculosis Control: Surveillance, Planning, Financing. WHO/HTM/TB/2005, Geneve, 349.

[6] Patel, A., Siskind, F.V., Abrahams, E. and Parker, J. (1991) Case-Control Evaluation of a School-Age BCG Vaccination Programme in Subtropical Australia. Bulletin of the World Health Organization, 69, 425-433.

[7] Zwerling, A., Shrestha, S. and David, W.D. (2015) Mathematical Modeling and Tuberculosis Advances in Diagnostics and Novel Therapies. Advances in Medicine, 2015, Article ID: 907267.

[8] Chavez, C.C. and Feng, Z. (1998) Global Stability of an Age-Structure Model for TB and Its Applications to Optimal Vaccination Strategies. Mathematical Biosciences, 151, 135-154. http://dx.doi.org/10.1016/S0025-5564(98)10016-0

[9] Chavez, C.C., Hethcotte, H.W., Andreasen, V., Levin, S.A. and Liu, W.M. (1989) Epidemi- 
ological Models with Age Structure, Proportionate Mixing, and Cross-Immunity. Journal of Mathematical Biology, 27, 233-258. http://dx.doi.org/10.1007/BF00275810

[10] Agusto, F.B., Cook, J., Shelton, P.D. and Wickers, M.G. (2015) Mathematical Model of MDR-TB and XDR-TB with Isolation and Lost to Follow-Up. Abstract and Applied Analysis, 2015, Article ID: 828461. http://dx.doi.org/10.1155/2015/828461

[11] Tewa, J.J., Bowong, S. and Mewoli, B. (2012) Mathematical Analysis of Two-Patch Model for the Dynamical Transmission of Tuberculosis. Applied Mathematical Modelling, 36, 2466-2485. http://dx.doi.org/10.1016/j.apm.2011.09.004

[12] Tewa, J.J., Bowong, S., Mewoli, B. and Kurths, J. (2011) Two-Patch Transmission of Tuberculosis. Mathematical Population Studies, 18, 189-205. http://dx.doi.org/10.1080/08898480.2011.596757

[13] Zhang, J. and Feng, G. (2015) Global Stability for a Tuberculosis Model with Isolation and Incomplete Treatment. Computation and Applied Mathematics, 34, 1237-1249. http://dx.doi.org/10.1007/s40314-014-0177-0

[14] Moualeu, D.P., Bowong, S., Tewa, J.J. and Emvudu, Y. (2012) Analysis of the Impact of Diabetes on the Dynamical Transmission of Tuberculosis. Mathematical Modelling of Natural Phenomena, 7, 117-146. http://dx.doi.org/10.1051/mmnp/20127309

[15] Greenhalgh, D. (1988) Threshold and Stability Results for an Epidemic Model with an AgeStructured Meeting Rate. IMA Journal of Mathematics Applied in Medicine and Biology, 5, 81-100. http://dx.doi.org/10.1093/imammb/5.2.81

[16] Dietz, K. and Schenzle, D. (1985) Proportionate Mixing Models for Age-Dependent Infection Transmission. Journal of Mathematical Biology, 22, 117-120. http://dx.doi.org/10.1007/BF00276550

[17] Zou, L., Ruan, S. and Zhang, W. (2010) An Age-Struvtured Model for the Transmission Dynamics of Hepatitis B. SIAM Journal on Applied Mathematics, 70, 3121-3139. http://dx.doi.org/10.1137/090777645

[18] Webb, G.F. (2008) Population Models Structured by Age, Size, and Spatial Position. In: Magal, P. and Ruan, S., Eds., Structured Population Models in Biology and Epidemiology, Springer-Verlag, Berlin, 1-49. http://dx.doi.org/10.1007/978-3-540-78273-5_1

[19] Inaba, H. (2006) Mathematical Analysis of an Age-Structured SIR Epidemic Model with Vertical Transmission. Discrete and Continuous Dynamical Systems Series B, 6, 69-96. http://dx.doi.org/10.3934/dcdsb.2006.6.69

[20] Djidjou, R.D., Tewa, J.J. and Bowong, S. (2014) Analysis of an Age-Structured SIL Model. With Demographics Process and Vertical Transmission. ARIMA Journal, 17, 23-52.

[21] Saylers, A.A. and Whitt, D.D. (1994) Bacterial Pathogenesis: A Molecular Approach. ASM, Washington DC. 
Submit or recommend next manuscript to SCIRP and we will provide best service for you:

Accepting pre-submission inquiries through Email, Facebook, LinkedIn, Twitter, etc. A wide selection of journals (inclusive of 9 subjects, more than 200 journals)

Providing 24-hour high-quality service

User-friendly online submission system

Fair and swift peer-review system

Efficient typesetting and proofreading procedure

Display of the result of downloads and visits, as well as the number of cited articles

Maximum dissemination of your research work

Submit your manuscript at: http://papersubmission.scirp.org/

Or contact am@scirp.org 